\title{
Design, Manufacturing, and Characterization of Hybrid Carbon/Hemp Sandwich Panels
}

\author{
Luca Boccarusso (D), Fulvio Pinto, Stefano Cuomo (D), Dario De Fazio, Kostas Myronidis, Massimo Durante, and Michele Meo
}

Submitted: 11 May 2021 / Revised: 21 July 2021 / Accepted: 20 August 2021 / Published online: 3 September 2021

\begin{abstract}
Advanced sandwich composite structures that incorporate foams or honeycombs as core materials, have been extensively investigated and used in various applications. One of the major limitations of the conventional materials used is their weak impact resistance and their end-of-life recyclability and overall sustainability. This paper is focused on the study of the production and mechanical characterization of hybrid sandwich panels using hemp bi-grid cores that were manufactured with an ad hoc continuous manufacturing process. Bi-grid structures were stratified in multiple layers, resulting in cores with different thicknesses and planar density. Sandwich panels made with carbon fibers skins were then subjected to Low Velocity Impact, compression and indentation and the damaged panels were investigated via CT-Scan. Results show that the high tailorability of the failure modes and the very good energy absorption properties of the hybrid material open new exciting perspectives for the development of new sandwich structures that can extend the use of natural fibers into several industrial applications.
\end{abstract}

Keywords CFRP, damage absorption, hemp, hybrid composites, LVI, natural fibers, sandwich

\section{Introduction}

Thanks to the excellent trade-off between strength and lightness, sandwich structures consisting of two relativity thin and high-performance skins divided by a thick and low-density core, are very popular in several application fields (Ref 1,2), such as aerospace, maritime, sports equipment, transportation, construction and defense (Ref 3,4).

In a typical sandwich panel, the skins act like the flanges in an I-beam carrying the main tensile and compressive loads, while the core acts like the web separating the load-bearing elements and increasing the moment of inertia; therefore the desired balance between mechanical properties and low weight can be obtained with any kind of material, both isotropic and anisotropic (Ref 5). In this context, it could seem that sandwich panels are not inherently connected with the use of composite materials; however since the use of fiber-reinforced laminates allows to further maximize the performance of these structures due to their high intrinsic mechanical properties, it is very common to find sandwich panels consisting of carbon, Kevlar or glass composite skins.

However, on one hand these materials confer excellent strength and high rigidity to the skins, on the other due to their

Luca Boccarusso, DarioDeFazio, and Massimo Durante, Department of Chemical, Materials and Production Engineering (DICMaPI), University of Naples Federico II, Piazzale V. Tecchio 80, 80125 Naples, Italy; and Fulvio Pinto, Stefano Cuomo, Kostas Myronidis, and Michele Meo, Department of Mechanical Engineering, Material Research Centre, University of Bath, Bath, UK. Contact e-mails: luca.boccarusso@unina.it, fp227@bath.ac.uk, sc2687@bath.ac.uk, dario.defazio@unina.it, km515@bath.ac.uk, mdurante@unina.it, and mm284@bath.ac.uk. low thickness and intrinsic laminated nature, do not offer any thermal or acoustic isolation and are characterized by low compression properties and weak impact resistance. As a consequence, the high in-plane properties of skins made of composites are usually coupled with the good compression properties and energy absorption of cores made of metallic, polymeric or natural materials. Furthermore, since the core has to be as light and thick as possible, its internal geometry also plays a fundamental role, leading to cores characterized by different material/geometry combinations which are available for specific applications.

According to the literature (Ref 6-9), cellular materials are frequently used as core for sandwich structures and can be classified according to the topology of their unit cell, as stochastic or periodic. The first category, also known as homogenous support cores (Ref 6), includes open and closed cell foams and is characterized by having random microstructures, while in the second core type, also known as nonhomogenous support core, the microstructures are periodic: either two-dimensional channels (honeycombs and prismatic materials) or three-dimensional truss or textile-based assemblies (Ref 7, 8, 10). Each of these categories offers some advantages and disadvantages and requires different manufacturing techniques; hence their selection strongly depends on the specific application requirements.

Based on this premise, it appears clear that the possibility to use hybrid composite materials can constitute a further push for the optimization of sandwich panels to a level which is unreachable when traditional metallic or polymeric materials are used. In particular, the development of hybrid panels characterized by a combination of two different reinforcements, guarantees higher tailorability of the structure, alleviating the inherent drawbacks of each of the different fibers while keeping the benefits of both, and at the same time enables synergistic effects that can lead to properties that neither of the constituents possesses, leading to the extension of the application fields for some materials that alone do not possess the required properties. 
A typical example of the latter is constituted by natural fiber composites (NFCs) that alone do not possess enough mechanical strength or rigidity to replace some high-performance composites like carbon fiber-reinforced polymers (CFRPs) but if adopted in a hybrid configuration with synthetic fibers can confer unique properties to the overall hybrid structure. This concept was emphasized by several researchers, for example Pinto et al. (Ref 11), Santulli et al. (Ref 12), Swolfs et al. (Ref 13) who proved that vegetable/carbon hybrid composites show improved mechanical properties and enhanced impact absorption performance if compared with traditional carbon fibers laminates due to the additional energy dissipation mechanisms given by the excellent damping properties of specific vegetable fibers, such as flax, hemp or kenaf.

It is also important to highlight that the expansion of the use of vegetables fibers into new technologies is in line with the current challenge to fight environmental issues allowing for the development of a new generation of composite systems characterized by low environmental impact, an efficient use of raw materials and the use of manufacturing processes with less environmental impact than traditional ones (Ref 14, 15).

It is therefore interesting to consider the use of NFCs as constitutive material for the core of hybrid sandwich structures, as evidenced by the numerous research works available in the literature.

For instance, Zuhri et al. (Ref 16) investigated the compression properties of square and triangular honeycomb core materials based on co-mingled flax fiber-reinforced polypropylene (PP) and polylactide (PLA) polymers and they were among the pioneers to study the potential application of natural fiber honeycomb cores for energy-absorbing structures and systems. The flax cores were characterized by a relative density in the range of $0.10-0.40 \mathrm{~g} / \mathrm{cm}^{3}$ and a thickness in the range of $0.9-2.00 \mathrm{~mm}$ and were produced adopting the non-continuous slotting procedure (i.e., stripes of composite laminates are interconnected through slots cut on their edges to obtain the final honeycomb structure). The results showed that these types of structures failed due to buckling phenomenon and that can reach a specific compression strength of around $25.46 \mathrm{kN} \mathrm{m} / \mathrm{kg}$ with a specific absorption energy up to around $6 \mathrm{~kJ} / \mathrm{kg}$.

Cicala et al. (Ref 17) studied the feasibility to use hemp/ epoxy bio-composites as base for an hexachiral auxetic trusscore for sandwiches produced by using the resin transfer molding (RTM) technique. They pointed out that the proposed hemp structure had a critical shear stress which was 32\% lower than the one reached for the same structure made in glass/ polyester but at the same time it showed a transverse shear modulus seven times higher. They also highlighted that biocomposite-based auxetic truss cores provided a weight saving of around $24 \%$ compared to polyester/glass structures making bio-composite structures very appealing.

Vitale et al. (Ref 18) pointed out that jute/epoxy honeycomb cores obtained by Vacuum Assisted Resin Transfer Molding (VARTM) can possess comparable flexural properties to the ones made with traditional materials (i.e., divinycell foam) and a good prediction of the mechanical behavior was obtained by developing an analytical model. Vitale et al. were one of the first to use natural fibers in form of fabric instead of single fibers to produce honeycomb core structures; however the adopted production strategy that mainly consists in the cutting of fabric strips and their placing between mold insert following a zigzag pattern is not continuous and not so easily implemented for an industrial production.
Sarwar et al. (Ref 19) were the first to design, fabricate, and test a hybrid sandwich Kevlar/Flax composite made from multiple layers of Kevlar fiber fabric for the skins and Flax fiber fabrics for the core. They produced the sandwich laminates by using the hand lay-up and compression molding techniques, and interesting results in terms of compression, tensile and torsion strength and low moisture absorption were achieved even though the manufacturing techniques adopted did not allow to obtain low density core.

Based on the results obtained in the research works cited above, it appears clear that there is a strong interest for the use of NFCs as core for sandwich panels; however at the same time it is clear that there are three main gaps that need to be filled before these materials can be fully exploited for these applications:

1. In order to employ hybrid natural composites for primary structures, an extensive investigation on the impact behavior of hybrid sandwich structures with NFC cores is required, as asserted by Chen et al. (Ref 20) especially under low-velocity impact conditions. Few works focused the attention on this topic by studying the impact behavior of hybrid sandwich structures, such as the work by Santulli et al. (Ref 12) who studied the energy absorption of sandwich panels made from woven carbon fibers (skins) and flax fibers (core) in an epoxy matrix. Even though the results from their experimental campaign are encouraging it must be pointed out that this paper does not focus on the optimization of the geometry of the NFC-based core; therefore the intrinsic lightness of these materials is not fully exploited and needs to be further investigated as also confirmed by some recent reviews (Ref 13, 21).

2. Since the majority of works available in the literature mainly focus the attention on the use of jute or flax as fiber for the production of hybrid sandwich (Ref 12, 13, $16,18,19,21,22)$, an extensive investigation on the possible use of others vegetable fibers as reinforcement for the development of new hybrid sandwich cores is required. In particular, due to their very good specific mechanical properties given by the high cellulose content and their excellent damping ability, hemp fibers constitute a valid candidate for hybrid sandwich cores especially when they are coupled with high strength carbon fibers, expanding their use from secondary to primary structures. In addition, considering that the environmental conditions required for the cultivation of hemp plants allow its easy growth around the world and its life cycle assessment (LCA) highlights further advantages over other vegetable fibers (Ref 23), these fibers are also characterized by low production cost.

3. As it is possible to see from many of the studies previously reported, one big limitation of the proposed hybrid solutions is constituted by the lack of a proper manufacturing technique that allows for the production of lightweight hybrid NF cores in a continuous way. Indeed, from the available literature, it is possible to note that all NFCs cores were made by adopting existing manufacturing process conventionally adopted for composite that cannot be scaled up easily for industrial production (i.e., the slotting method (Ref 16, 24), or techniques that require long time for the mold preparation and for the fibers positioning, such as RTM (Ref 17, 18). 
In a work done by the same authors (Ref 25) the manufacturing process of NF cores to produce sandwich panels in a continuous way was presented and some initial insight on their impact behavior were offered. Being a proof of concept of the manufacturing process, the work did not fully investigate the feasibility of the manufacturing technique and in particular did not focus on the possibility to produce cores with different thicknesses the effect that thickness had on the failure mechanisms of the hybrid panels. The present work represents the natural progression of Ref 25 and is aimed at filling the above said gaps by fully analyzing the capabilities of the manufacturing technique and offers a complete characterization of the proposed hybrid sandwich panel. Indeed, the influence of the thickness of the core on the impact performance of the sandwich panels is analyzed at different energetic levels (10, 25,45 and $50 \mathrm{~J}$ ) and indentation and flatwise compression tests are performed on the hemp cores with different densities to characterize their failure modes and energy dissipation ability.

\section{Materials and Methods}

\subsection{Materials}

Sandwich structures under investigation consist of carbon/ epoxy skins and two hemp/epoxy core types that differ in their density. In detail, two types of woven hemp fabrics (Fidia Srl) having areal densities (GMS) of 380 and $190 \mathrm{~g} / \mathrm{m}^{2}$, labeled HD and $\mathrm{LD}$, respectively, and an SX10 epoxy resin (Mates Srl) were used to manufacture the hemp cores. The HD and LD fabrics differ in the number of fibers' intersection and then in the number of tows in warp and weft direction. A schematization of their elementary cell (Fig. 1) highlights the main geometrical properties of the two grid hemp fabrics.

In order to manufacture the carbon skins, a XPREG XC110 (EasyComposites) carbon fabric prepreg with an areal density of $210 \mathrm{~g} / \mathrm{m}^{2}$ was used and an epoxy adhesive film (XPREG XA120, Easy Composites) was applied between core and skins for the sandwich assembling.

\subsection{Core Design}

The geometry of the core structure represents a key parameter in the optimization of the performance of a sandwich panel; therefore the core structure was designed following two guidelines: (1) the weight of the core needs to be as light as possible without sacrificing the adhesion with the skin (i.e., good shear properties) and (2) the hemp fibers need to be oriented in order to optimize the out-of-plane properties of the panel, and in particular improve impact resistance and energy absorption. Based on these premises, together with the need of an ad hoc manufacturing technique able to be scaled-up for industrial production, the NFC core was manufactured by using a series of grid layers made with a planar mesh of hemp tows.

Grids are planar structures characterized by the presence of ribs that run in several directions for multidirectional loadings and are usually classified as bi-grids, tri-grids and quad-grids according to their orientation. Among them bi-grids are simpler to manufacture due to the lower number of rib-crossings at the intersections that reduces the complexity of the weaving process but their compression strength is usually low due to the occurrence of buckling phenomena. To overcome this limitation while keeping the advantage of a simple manufac- turing process, the NF core of this work is manufactured by overlapping different fibrous bi-grids layers and impregnating them with resin in order to form a solid three-dimensional grid structure. To the authors' knowledge this is the first attempt to manufacture a NF core using planar grids. The only work found by the authors concerning the use of NF fabrics as core for hybrid sandwiches is the one of Sarwar et al. (Ref 19). However, in this work the authors produced hybrid sandwich structures by using hand lay-up and compression molding techniques without focusing the attention on the density of the core and on the mesh size of the fabric; therefore their final core structure was not an open cell grid. The presence of multilayered structures allows for the use of bi-grid layers characterized by a large mesh size, reducing the overall core weight and simplifying the resin removal during the manufacturing process.

Two fabric typologies were manufactured by changing the areal density in order to produce a low density hemp layer (LD) and a high density one (HD). Both LD and HD fabrics were used to manufacture the three-dimensional grids

\subsection{Sandwich Manufacturing}

Before the impregnation phase, in order to improve the adhesion with the epoxy matrix, the hemp fabric was soaked in $2 \% \mathrm{NaOH}$ solution at room temperature for $30 \mathrm{~min}$, copiously washed with water to remove any traces of alkali on the fibers surface and subsequently neutralized with $1 \%$ acetic acid solution. Then, the treated fibers were dried in an oven at $60{ }^{\circ} \mathrm{C}$ for $12 \mathrm{~h}(\operatorname{Ref} 23,26)$.

The production system used to produce the bio-based hemp core (Ref 25) is schematized in Fig. 2 and it is divided in the following steps:

- Impregnation

- Resin content reduction

- Resin absorption

- Cutting

- Polymerization

In the first step, a caterpillar apparatus localized at the end of the production system pulls and forces the hemp fabric in a resin bath, where tension rolls squeeze the fibers in order to obtain a good impregnation.

At this point, the impregnated fabric passes under an air jet flow that blows through the mesh of the fabric and then, between a couple of rolls covered with absorbent paper layers; thanks to the large mesh that characterizes the bi-grid layer, the air flow is able to remove the excess of resin between the spaces reaching the desired fiber content. By controlling the amount of resin in the resin bath and by tailoring the resin removal process, the ratio between the weight of the impregnated and unimpregnated layers was kept constant to 2.5 and the fiber weight fraction content of each layer at almost $40 \%$.

Finally, the impregnated fabric is cut in layers $100 \times 150 \mathrm{~mm}^{2}$ in dimension and each layer is stacked one on the other in a steel plate mold. When the desired number of layers is stacked together, the mold was closed by using a polymeric bag and a vacuum of 0.9 bar was applied by using a pump. Continuing the application of the vacuum, the closed mold is placed in a hydraulic press applying an additional pressure of 1 bar to allow the cure of the laminate at room temperature for $24 \mathrm{~h}$. 


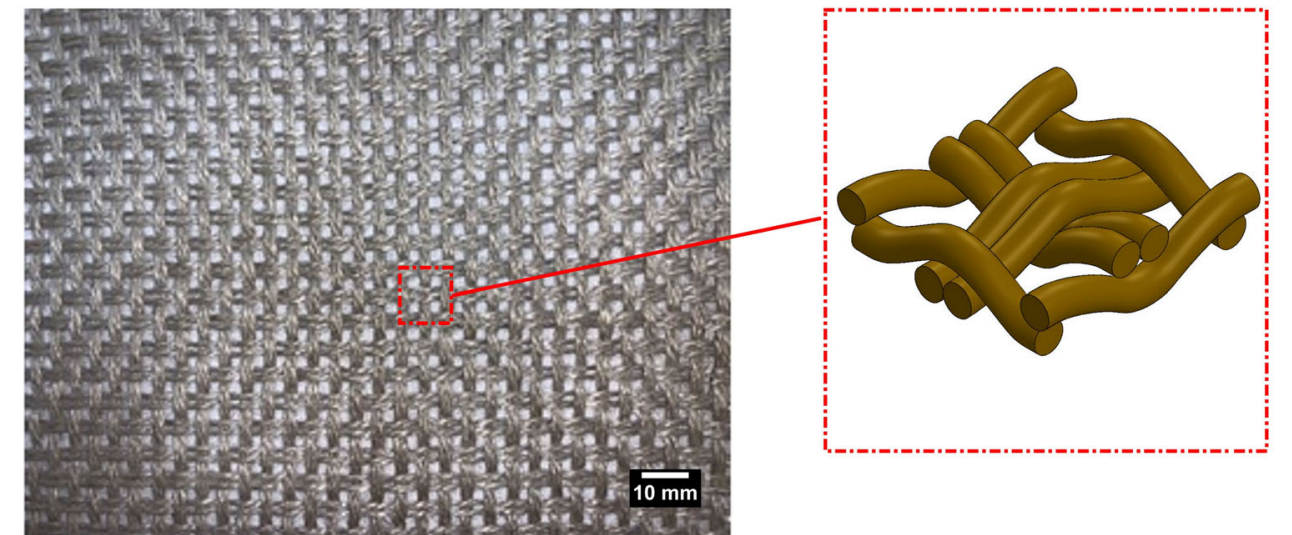

(a)

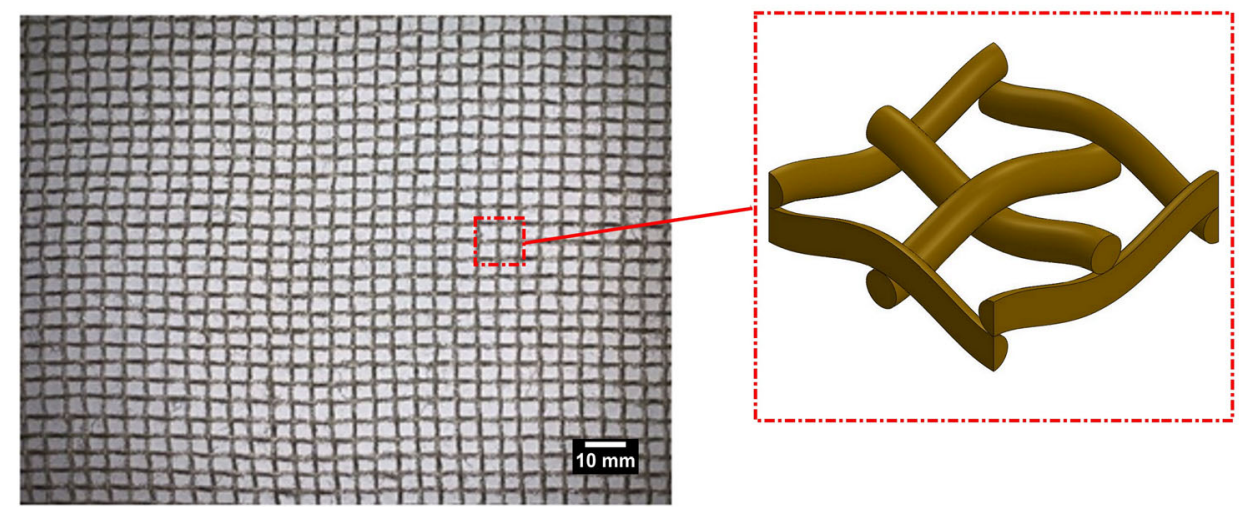

(b)

Fig. 1 Woven hemp fabric and schematization of their elementary cell for (a) HD and (b) LD type

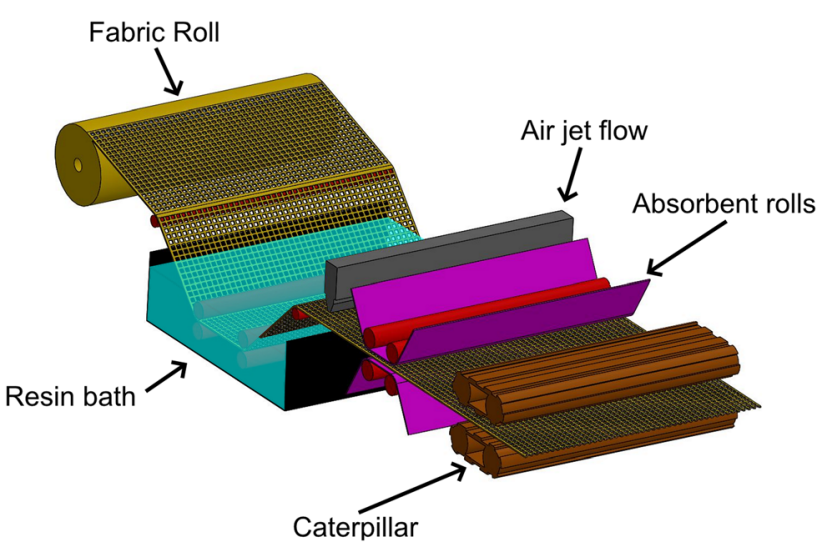

Fig. 2 Schematization of the production system

To produce cores with different thicknesses, samples with 2, 4 and 6 layers of bi-grid hemp fabrics were manufactured.

The carbon fiber laminates were manufactured by layering 4 prepreg plies and curing them in autoclave with a pressure of 8 bar at $120{ }^{\circ} \mathrm{C}$ for $8 \mathrm{~h}$. At the end of the polymerization phase, the CFRP laminates were cut to plates measuring $100 \times 150 \times 1 \mathrm{~mm}^{3}$.

The sandwich panels were then assembled by placing a single layer of epoxy adhesive film between the carbon skins and the hemp core, and then the panels were sealed in an elastomeric bag under vacuum and cured with a pressure of 8 bar at $120{ }^{\circ} \mathrm{C}$ for $8 \mathrm{~h}$.

In order to investigate both the effect of the thickness of the core and the density of the bi-grid layer, six different sandwich typologies were manufactured by using 2, 4 or 6 layers of LD or HD hemp layers. The specific of each sample's typology are summarized in Table 1.

\section{Experimental Campaign}

The experimental campaign was based on the hypothesis that the response of the NF cores to an external stimulus would be strongly affected by their density. In particular, despite the intrinsic damping characteristics of the hemp fibers, the samples will respond to an out-of-plane load with a more brittle or pseudo-plastic behavior, according to the number of hemp tows that constitute the unit cell.

In order to support this, the response of a sample of a specific thickness from both LD and HD configurations was recorded for impacts at different energy levels; this enabled an accurate understanding of how the different samples absorbed and dissipated energy and what failure mechanisms emerged. Then, the effect of the thickness was analyzed by adding layers of the grid structure to the NF cores and testing the response to a given energetic level while also measuring the difference in the response of the LD and HD typologies. CT-Scan analysis was used to reveal the different failure modes and damage 
Table 1 Main properties of all specimens under inspection

\begin{tabular}{lcccccc}
\hline Label & Number of core plies & Weight, $\mathbf{g}$ & Sandwich thickness, mm & Core thickness, mm & Core density, g/cm & Fiber weight, $\%$ \\
\hline HD_6P & 6 & 81.46 & 9.93 & 8.16 & 0.63 & 40 \\
HD_4P & 4 & 55.12 & 7.32 & 5.54 & 0.65 & 40 \\
HD_2P & 2 & 26.24 & 4.66 & 7.84 & 0.60 & 47 \\
LD_6P & 6 & 39.25 & 8.76 & 4.88 & 0.37 & 40 \\
LD_4P & 4 & 26.88 & 6.64 & 2.66 & 0.33 & 40 \\
LD_2P & 2 & 13.28 & 4.41 & & \\
\hline
\end{tabular}

propagation mechanisms and to determine any similarities or differences between the two typologies. Considering the dynamic evolution of the impact force during the LVI, an indentation test was selected to provide results under controlled displacement conditions of the indenter, in order to further corroborate the results of the LVI campaign. Finally, the flatwise compression test yielded the compression strength of the NFC cores.

\subsection{Low Velocity Impact Test}

The impact test campaign was carried out using a drop weight tower apparatus, characterized by a $9 \mathrm{~kg}$ shuttle moving on low friction guides and a $15 \mathrm{~mm}$ hard steel hemispherical tip. The rig was equipped with an anti-rebound system to avoid the occurrence of multiple impacts on the tested specimen; hence the response of one single impact was acquired by a load cell mounted on the shuttle; data from the impact event, via a digital oscilloscope were then stored in a pc.

The experimental campaign was performed in accordance with ASTM D7136 standard; hence rectangular specimens of $100 \times 150 \mathrm{~mm}^{2}$ dimensions were tested. In order to avoid vibrations and motions during the impact a specific fixture was used to clamp the specimens.

Four different energies were tested during the experimental campaign that can be summarized as follows and for each configuration three samples were tested:

- $10 \mathrm{~J}$ (2 and 4 plies HD and LD)

- $25 \mathrm{~J}(2,4$ and 6 plies HD and LD)

- $45 \mathrm{~J}(2,4$ and 6 plies HD and LD)

- $50 \mathrm{~J}$ (6 plies HD and LD)

All the energy levels were obtained changing the drop height by which the shuttle was released.

\subsection{Non-destructive Tests: Computed Tomography (CT) Scan}

The equipment used was a Nikon XT H 225 for the image acquisition and the analysis was carried out following the ASTM E1672 standard.

The inspection process was performed acquiring the top and the bottom view and two cross sections, on transversal and on longitudinal direction, of each tested specimen.

Avizo Software was used to perform the post-processing of the pictures.

\subsection{Indentation Test}

The indentation test was carried out with a MTS Alliance $\mathrm{RT} / 50$ universal testing machine equipped with a $50 \mathrm{kN}$ piezoelectric load cell and a $15 \mathrm{~mm}$ hard steel hemispherical tip indenter in order to simulate the force-displacement relationship during the impact event. The indentation tests were carried out on both the HD 6P and the LD 6P hemp core samples and a total of three tests were performed for each typology: at penetration, at $33 \%$ and at $66 \%$ of the maximum displacement obtained at penetration. The testing speed was set to $4 \mathrm{~mm} / \mathrm{min}$ according to the ASTM D6264 standard, as well as the fixture geometry (steel plate with a $125 \mathrm{~mm}$ open hole) and the specimens' dimension $\left(150 \times 150 \mathrm{~mm}^{2}\right.$ plates with 6 layers core).

\subsection{Compression Test}

Flatwise compression tests were carried out using the same testing machine adopted for the indentation tests. As well as the indentation, this test was carried out on both the LD 6P and the HD 6P hemp core samples according to ASTM C365 standard. The compression tests were carried out with a testing speed of $4 \mathrm{~mm} / \mathrm{min}$ on 6 layers square core samples $21 \times 21 \mathrm{~mm}^{2}$ in dimension, characterized by 10 and 5 tows, respectively, for the HD and LD samples on both warp and weft direction.

Once the compression force and the crosshead displacement were acquired, the compression strength $\left(\sigma_{\mathrm{c}}\right)$ was evaluated using the following equation.

$\sigma_{\mathrm{c}}=\frac{F}{A}$

where $(F)$ is the force in $N$ measured in correspondence of the load deflection of the compression curve according to the standard's procedure, $(A)$ is the effective horizontal cross section obtained by considering the effective number of core tows in warp and weft direction. The compression modulus was evaluated by considering the stress to strain ratio.

\section{Results}

\subsection{Impact Tests}

Figure 3 shows typical force vs. displacement curves related to the LD 4P samples impacted at three different energy levels $(10,25$ and $45 \mathrm{~J})$; a cross section in the transverse direction is represented next to the curves, for a visual representation of the damage extent within the samples. Figure 4 includes the corresponding curves and images for the HD_4P.

Analyzing the force-displacement curves of LD samples (Fig. 3), the impacts carried out up to $25 \mathrm{~J}$ are characterized by a typical elastic behavior, clearly showing the rebound of the impactor tip without any load drops. Increasing the energy to $45 \mathrm{~J}$, around the maximum displacement value reached for $25 \mathrm{~J}$ 

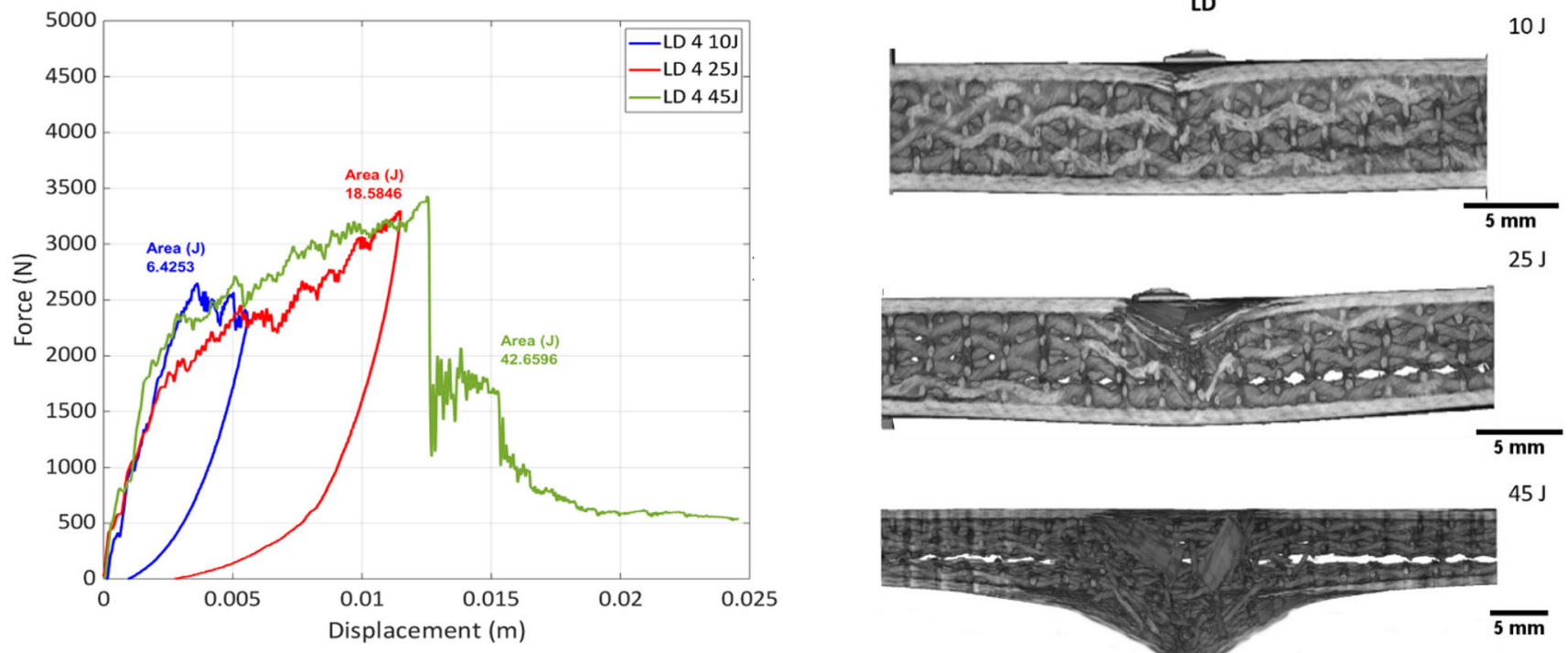

$45 \mathrm{~J}$

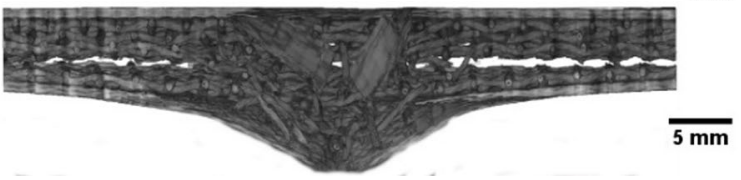

Fig. 3 Impact curves (force versus displacement) for LD typology at different energy level with the same number of plies (four) and corresponding images from CT-scan for each specimen
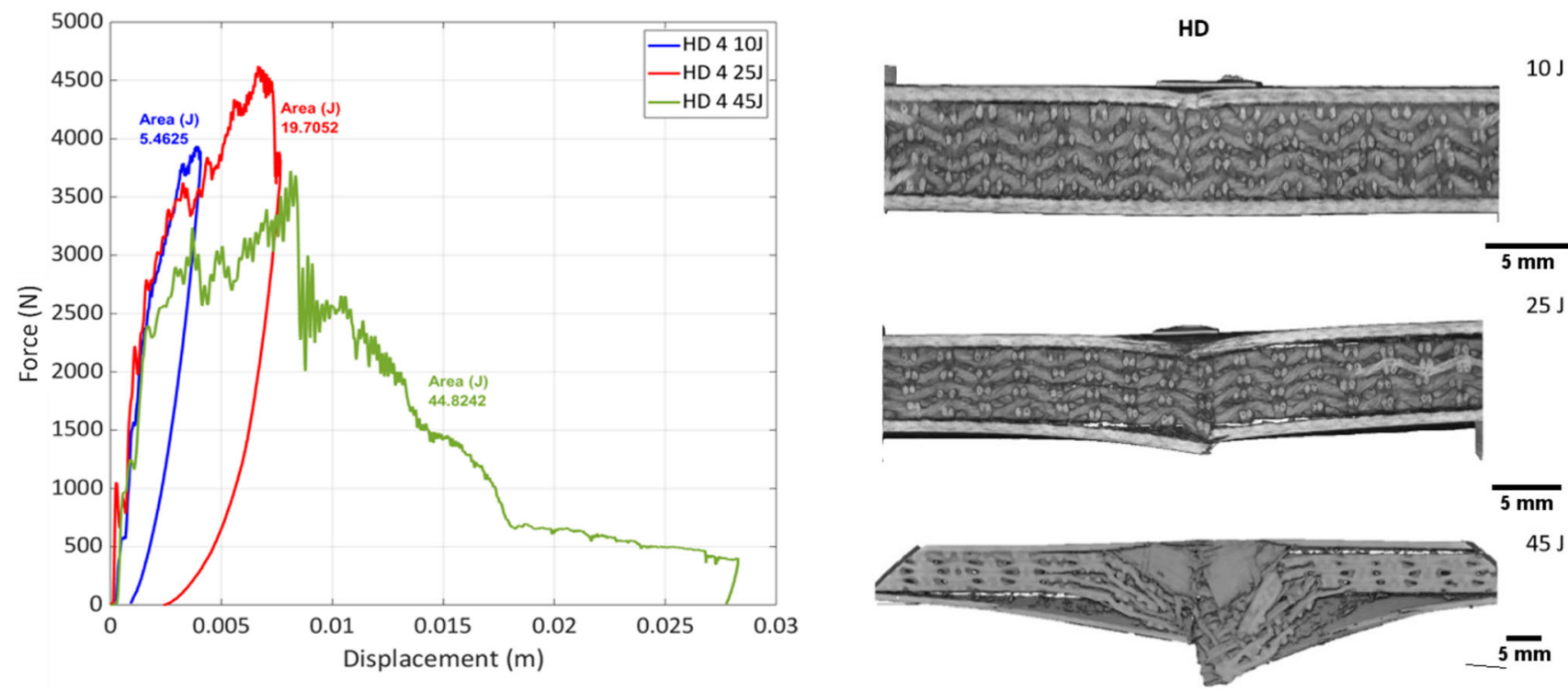

Fig. 4 Impact curves (force versus displacement) for HD typology at different energy level with the same number of plies (four) and corresponding images from CT-scan for each specimen

there is a clear load drop, a sign of the onset of damage in correspondence with the bottom skin. The response of all the three samples during the first instants of the impact is consistent, with similar level of stiffness. At $45 \mathrm{~J}$ higher force values are reached faster than previous energy levels, inducing higher stress level and consequent damage.

The effects of different energy levels on the same HD_4P samples are comparable to the results discussed above. Increasing the energy from 10 to $25 \mathrm{~J}$ the trend is similar, with highest peak force and displacement values in the latter. The impact behavior changes at $45 \mathrm{~J}$ with lower peak force $(-22 \%)$ and clear signs of damage (load drop) and residual elastic behavior. In terms of stiffness, during the first moments of the impact all three samples show similar response to the dynamic load.

Comparing the curves at different energy levels, it is possible to note that HD_4P specimens show a stiffer response compared to the LD $4 \mathrm{P}$ ones. This behavior is expected since the HD samples are characterized with a denser hemp core, with double fibers per unit of weight compared to the LD samples. The density of the core as well as the geometric parameters, are parameters that directly affect the impact behavior of the whole sandwich as stated in (Ref 27).

At $10 \mathrm{~J}$ the HD configuration shows almost pure elastic behavior, while the LD undergoes some plastic deformations with higher absorbed energy given by the formation of new surfaces due to the internal cracks and delamination. An 
interesting insight is given by the comparison of the maximum peak forces; indeed, the LD_4P samples show a reduced peak force $(-32 \%)$ compared to the HD_4P specimens, and the same trend is detected increasing the impact energy at $25 \mathrm{~J}$, with a reduced peak force $(-28 \%)$. At $45 \mathrm{~J}$ the maximum peak force level reached for both typologies is almost comparable ( $-8 \%$ by LD compared to HD).

At $25 \mathrm{~J}$ the response of LD_4P and HD_4P samples is similar, with the latter characterized by a narrow forcedisplacement curve with an almost elastic response, while the former shows higher displacement. At $45 \mathrm{~J}$ the damage threshold is reached, with a clear drop in the force-displacement curve for both specimen types. In particular, the HD samples present a drop at $3685 \mathrm{~N}$ and $8.34 \mathrm{~mm}$, showing a residual elastic behavior (meaning that a small rebound of the impactor occurred), in contrast to the LD samples that dropped at a similar value of the force $(3431 \mathrm{~N})$ but at a higher value of displacement $(12.52 \mathrm{~mm})$ showing the typical response when the tip of the impactor is fully penetrated inside the sample (Ref 28). Figure 5 shows the average values of absorbed energy per unit of weight at different energy levels for both LD_4P and HD_4P samples with same core thickness.

As displayed in Fig. 5, increasing the impact energy, the absorbed energy per unit of weight increases for both LD and HD families. At the same energy level the absorbed energy per unit of weight is higher for LD type compared to HD one, with an increase of $+50,+35,+28 \%$ at 10,25 and $45 \mathrm{~J}$, respectively. Hence, considering the same core sandwich (4 plies hemp core), at different energy levels, LD 4P samples show an increased absorbed energy per unit of weight and a reduced maximum peak force compared to the $\mathrm{HD}$ 4 $\mathrm{P}$ ones.

Figure 6 reports the comparison between samples made with three different LD core thicknesses (2, 4 and 6 plies), impacted at $25 \mathrm{~J}$ and the corresponding images obtained from CTscanning in the transverse plane. Figure 7 includes the analogous curves and images for the HD typology.

The 2 plies samples show a similar behavior for both LD and HD types, with a clear drop in the force when the damage threshold is reached. It is important to highlight that the maximum peak force for LD type is reduced by $21 \%$ compared to $\mathrm{HD}$, with a higher displacement $(+58 \%)$ experienced by the former. This behavior is consistent with the stiffness properties of the samples, where the LD being able to displace more than the HD is then affected by a reduced load peak.

The samples with 4 plies show similar response, showing also in this case a lower peak force $(-28 \%)$ for the LD

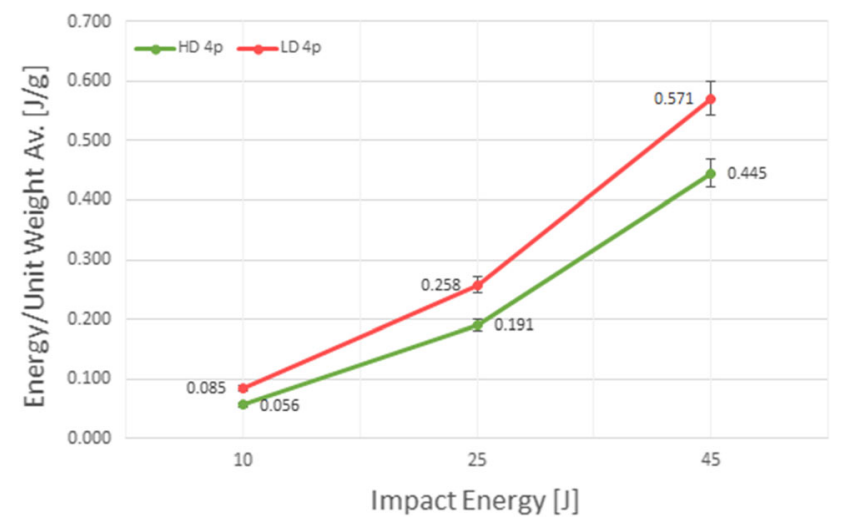

Fig. 5 Energy absorbed per unit of weight for each energy level samples compared to the HD ones. The HD specimens show a drop in the load curve, sign of onset damage, just before the maximum displacement is reached with a typical behavior that has been already reported in the literature (Ref 29).

The energy absorbed by the 6 plies LD specimen is $23 \%$ higher than the HD. Comparing the curves of the two sample types the difference in response is evident, with a reduced peak force of approximately $36 \%$ for the LD configuration compared to the HD one. All the curves in Fig. 6 and 7 show a very clear drop in the first instances of the impact due to cracking and damage of the outer skins of the sandwiches.

The bar plot, which reports the absorbed energy per unit of weight for the impact tests performed at $25 \mathrm{~J}$ (Fig. 8), confirms the trend observed in the previous comparison (see Fig. 5), with higher absorbed energy per unit of weight experienced by the LD type compared to the HD. Increasing the number of plies, the absorbed energy per unit weight at same impact energy is reduced as the same amount of load is distributed on a higher volume, while the force increases.

The effect of increasing the core thickness on the impact performance of the LD sandwich is appreciable from Fig. 8. Increasing the thickness, the absorbed energy per unit of weight is reduced $(-28 \%)$ for both 4 and 6 plies cores compared to the 2 plies one. Hence, an increase in core dimensions is not linked to an improvement of impact energy absorption performance.

The same trend is confirmed with the HD sandwich type. Increasing the core thickness from 2 to 4 and 6 plies, the absorbed energy per unit of weight is reduced by $39 \%$ and $53 \%$, respectively.

An interesting insight is the comparison between the LD and HD impact absorption performance. Overall, as mentioned before, both families show a reduction in absorbed energy increasing the core thickness, but the LD type is affected by lower peak force $(-36 \%, 6$ plies) and higher absorbed energy per unit of weight $(+72 \%, 6$ plies) when is compared to similar HD type. Hence, the LD samples are characterized by better energy absorption capability, showing reduced level of load during the impact event.

In order to evaluate the level of energy by which the thicker samples are subjected to perforation, both the LD_6P and HD_6P samples were subjected to impacts at 45 and $\overline{50} \mathrm{~J}$. As depicted in Fig. 9 the LD samples still do not show signs of complete perforation when impacted at $45 \mathrm{~J}$, while this is evident when the energy is raised to $50 \mathrm{~J}$ (see Fig. 9). For both the 45 and $50 \mathrm{~J}$ impacts, the impact responses of the HD_6P samples (see Fig. 10) show a stiffer behavior compared to the LD_6P ones, with a higher peak force $(+20$ and $+37 \%)$ and a reduced maximum displacement $(-38$ and $-70 \%)$. In particular, the LD_6P samples show a first load drop in correspondence with the failure of the top skin, followed by an increase in the force given by the crushing of the core, until the second drop that corresponds to the failure of the lower skin. On the contrary, for both the energies the HD_6P samples show only the drop due to the top skin failure and then the rebound, with core crushing concentrated on top and delamination of the lower layers, with onset damage of the outer skin at $50 \mathrm{~J}$.

The higher energy absorption performance of the LD samples is confirmed also for the impacts at 45 and $50 \mathrm{~J}$, with an increase of $59 \%$ in energy absorbed per unit weight for the LD samples $(0.57 \mathrm{~J} / \mathrm{g})$ compared to $\mathrm{HD}(0.35 \mathrm{~J} / \mathrm{g})$.

The analysis of the results above, makes evident the difference in the behavior of the two proposed cores, as the 

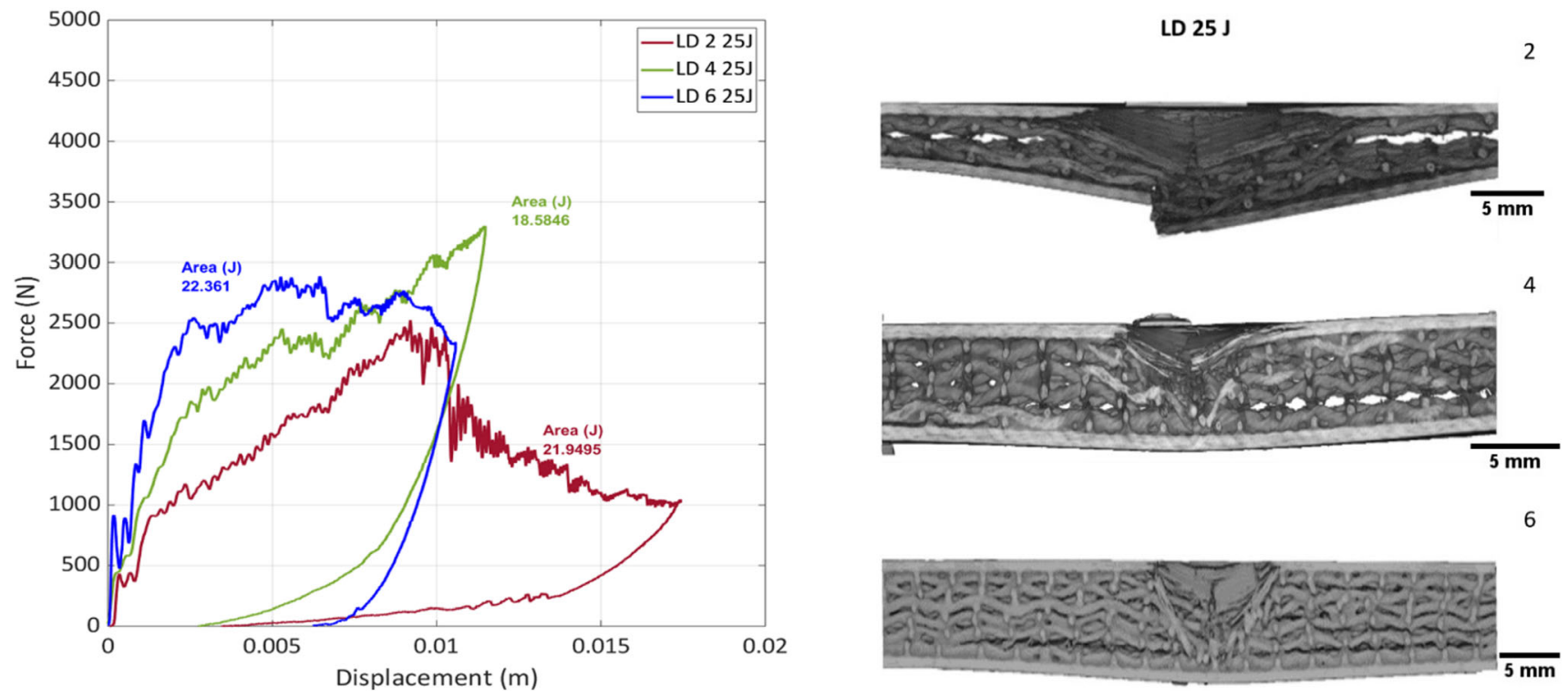

6

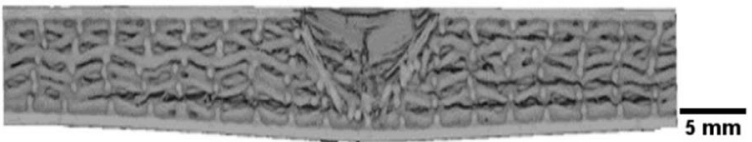

Fig. 6 Impact curves (force versus displacement) for LD typology at same energy level and different number of plies and corresponding images from CT-scan for each specimen

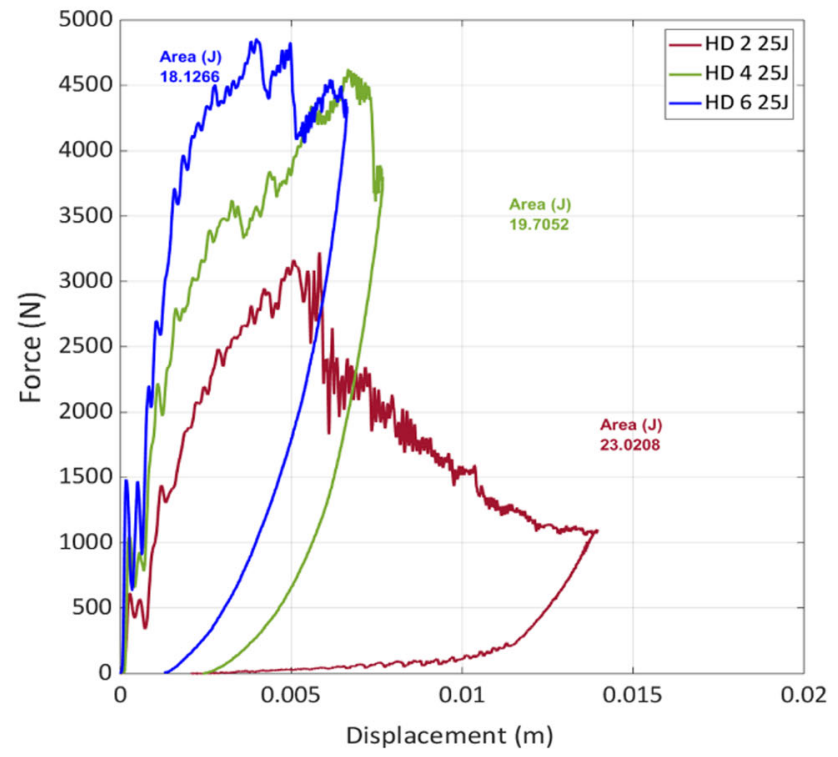

HD 25 J

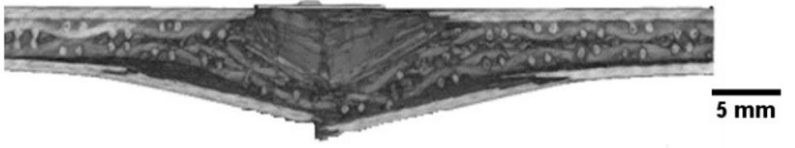

4

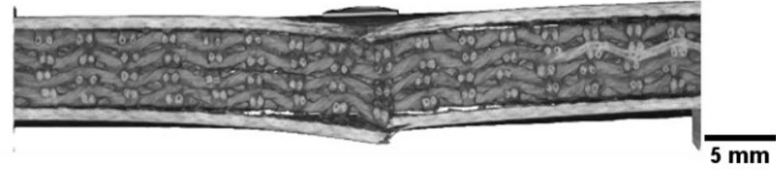

6

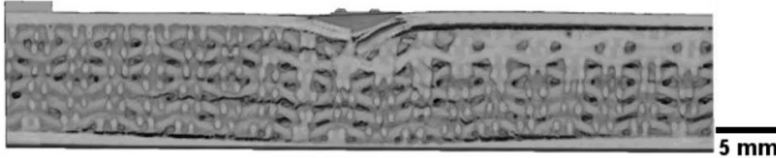

Fig. 7 Impact curves (force versus displacement) for HD typology at same energy level and different number of plies and corresponding images from CT-scan for each specimen

LD samples are able to absorb more impact energy per unit of weight than the HD ones. It is important to note that increasing the thickness of the core resulted in an increase in the absorbed energy for both typologies; however this increase is more efficient for the LD samples which showed a maximum increment of $+70 \%$ when six plies are considered. The higher energy absorption performance of the LD samples was also confirmed at 45 and $50 \mathrm{~J}$.

\subsection{CT-Scan Test}

In order to investigate the effect of the impact energy on a given thickness and compare to failure modes and sequences of failure events from the literature (Ref 30) post-impact samples were analyzed via CT-scan. Figure 11 depicts the comparison of both LD_4P and HD_4P samples impacted at different energy levels, 10-25-45 $\overline{\mathrm{J}}$, respectively. The LD typology impacted at $10 \mathrm{~J}$ shows a clear damage on the top skin and tow failure inside the core. The propagation of the crack from the face sheet, to the face sheet-core interface and inside the core is particularly evident. The HD typology revealed a noteworthy difference in the energy dissipation mechanism between the two typologies; after the initial delamination of the top face sheet (significantly smaller than that for the LD one), shear stresses between the face sheet and the face sheet-core region prevailed, leading to the onset of skin-core debonding.

The same trend is visible in the specimens impacted at $25 \mathrm{~J}$, however, more amplified due to the increased impact energy levels. For the LD typology, the damage has evolved through the thickness of the sample, in a region close to the impacted 
area, leading to delamination in the impacted face sheet, core crushing, matrix cracking and breakages on the fibers in the top facing and the core. Another notable characteristic of the LD_4P sample is the appearance of an intra-core delamination. The HD_4P sample revealed top face sheet failure, increased debonding in the area between the top skin and the core, tow failure in the core and finally bottom face sheet failure in the form of fiber breakage. At $45 \mathrm{~J}$ and despite the great amount of impact energy the samples were subjected to, the same failure mechanisms of the samples are detected. Both face sheets of the LD_4P sample are damaged and intra-core delamination and core crushing is evident. The HD typology core crushing is owed to the aforementioned failure modes.

The different response of the two types of specimens, can be attributed to the increased number of contact points between the two-unit cells of the samples, as illustrated in Fig. 12.

At the center of the LD typology's unit cell, a single contact point leads to bending during impact; consequently, the response of the whole structure is more elastic. The antisymmetric wrinkling (Ref 31) of the sandwich composite structure

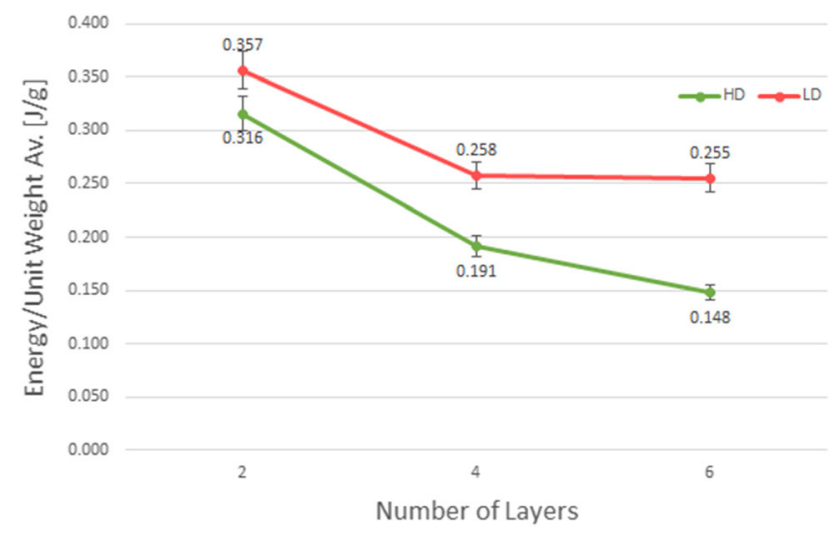

Fig. 8 Plot of the energy absorbed per unit of weight for each sample type results in intra-core delamination. On the contrary, the additional contact points of the HD typology, result in a stiffer response of the structure. This leads to increased shear stresses at the face sheet-core interface with the energy dissipating through the thickness of the core, leading to bottom face sheet failure.

Figure 13 depicts the comparison between the LD and HD samples when impacted at $25 \mathrm{~J}$ and illustrates the effect of thickness on both sample families. In particular, the LD_2P specimen shows clear sign of delamination localized in the mid plane between top and bottom layers, while the HD_2P does not show intra-core delamination showing instead partial debonding of the bottom skin. In order to focus more in detail on the interfacial failure of sample HD_2P (illustrated in Fig. 14a and b) the skin of the sample represented in Fig. 7 was manually removed by the core without damaging the area surrounding the debonded surface and subjected first to a visual inspection and then to a more in depth analysis using a Leica Optical Microscope. Analyzing the results, it was possible to observe traces of adhesive on both the core and the skin, suggesting cohesive failure. The regular pattern of the adhesive is in fact present in correspondence with the points of contact between the core and the skin, as evident by Fig. 14(c) and (d). Microscope images of the areas surrounding the back crack showing clearly the presence of the adhesive both on the surface of the skin than on the hemp yarn that forms the core, as illustrated in Fig. 14(e) and (f). The cohesive failure is further suggested by the fact that when the skin was removed, part of the hemp core remained attached to the skin indicating that the crack propagated through the core in the areas surrounding the cracks close to the point of contact, as it is possible to see from Fig. 14(g). It must be noted that the higher density of the HD layers allows for a number of points of contact between the core and the skins which is quadruple in respect with the LD cores; therefore the adhesion between the HD core and the CFRP skin is stronger than the one of the LD panels. The fact that the core/ skin debonding failure is evident only the HD samples suggests that phenomenon is therefore not the result of poor adhesion but it is caused by the increased amount of energy available for

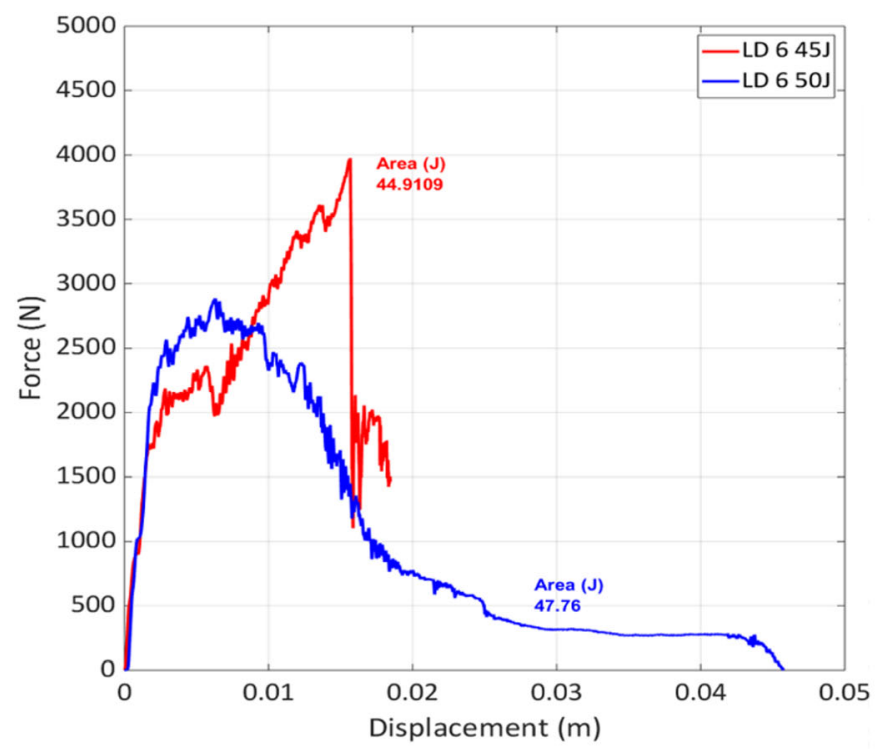

LD

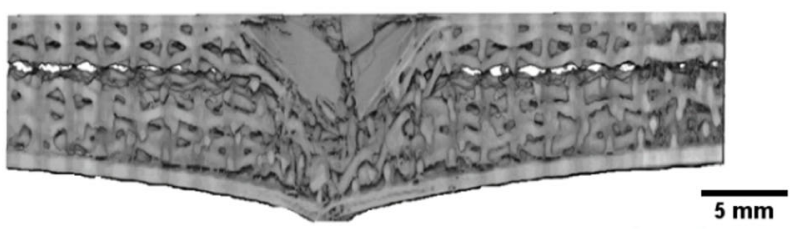

$50 \mathrm{~J}$

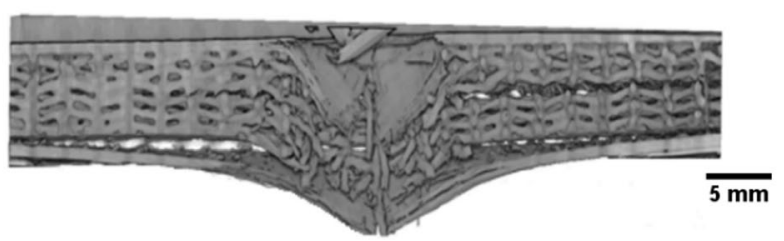

Fig. 9 Impact curves (force versus displacement) at 45 and $50 \mathrm{~J}$ energy levels for LD_6P typology and corresponding images from CT-scan for each specimen 

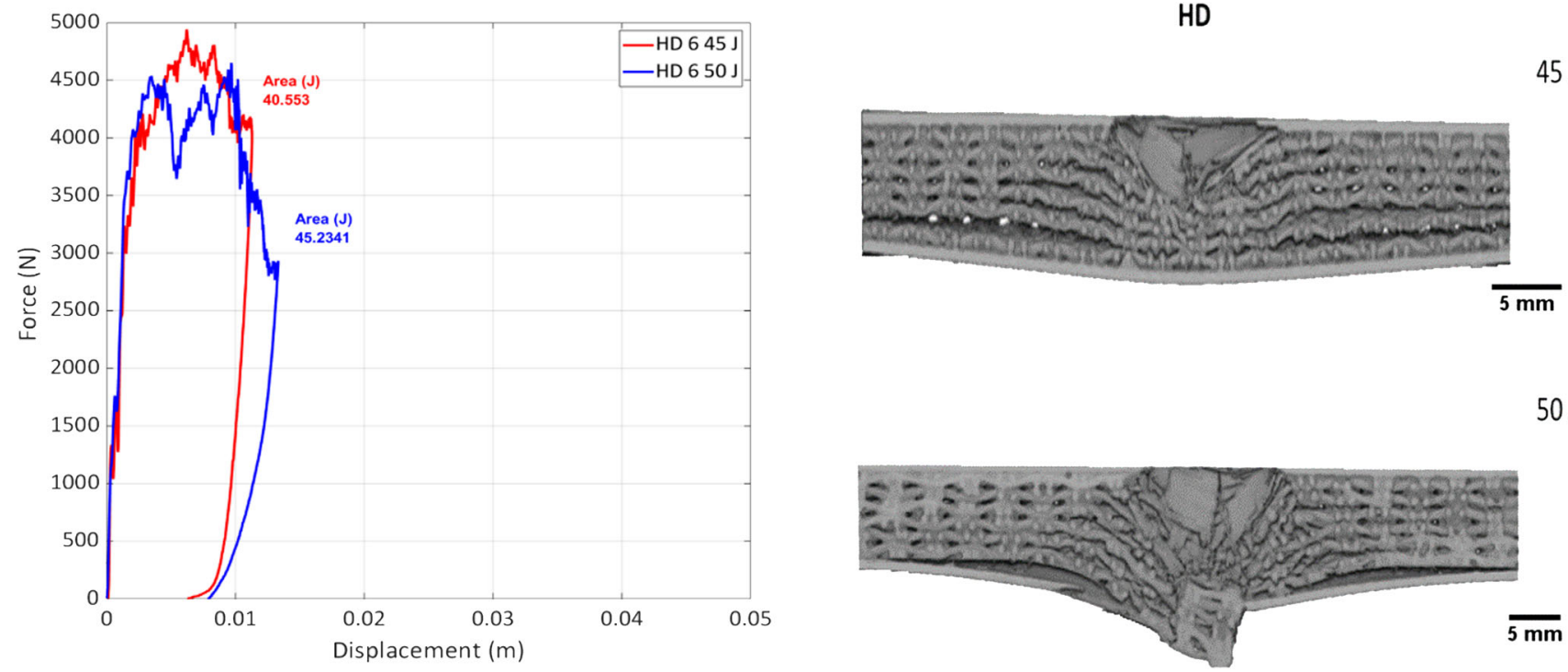

Fig. 10 Impact curves (force versus displacement) at 45 and $50 \mathrm{~J}$ energy levels for HD_6P typology and corresponding images from CT-scan for each specimen

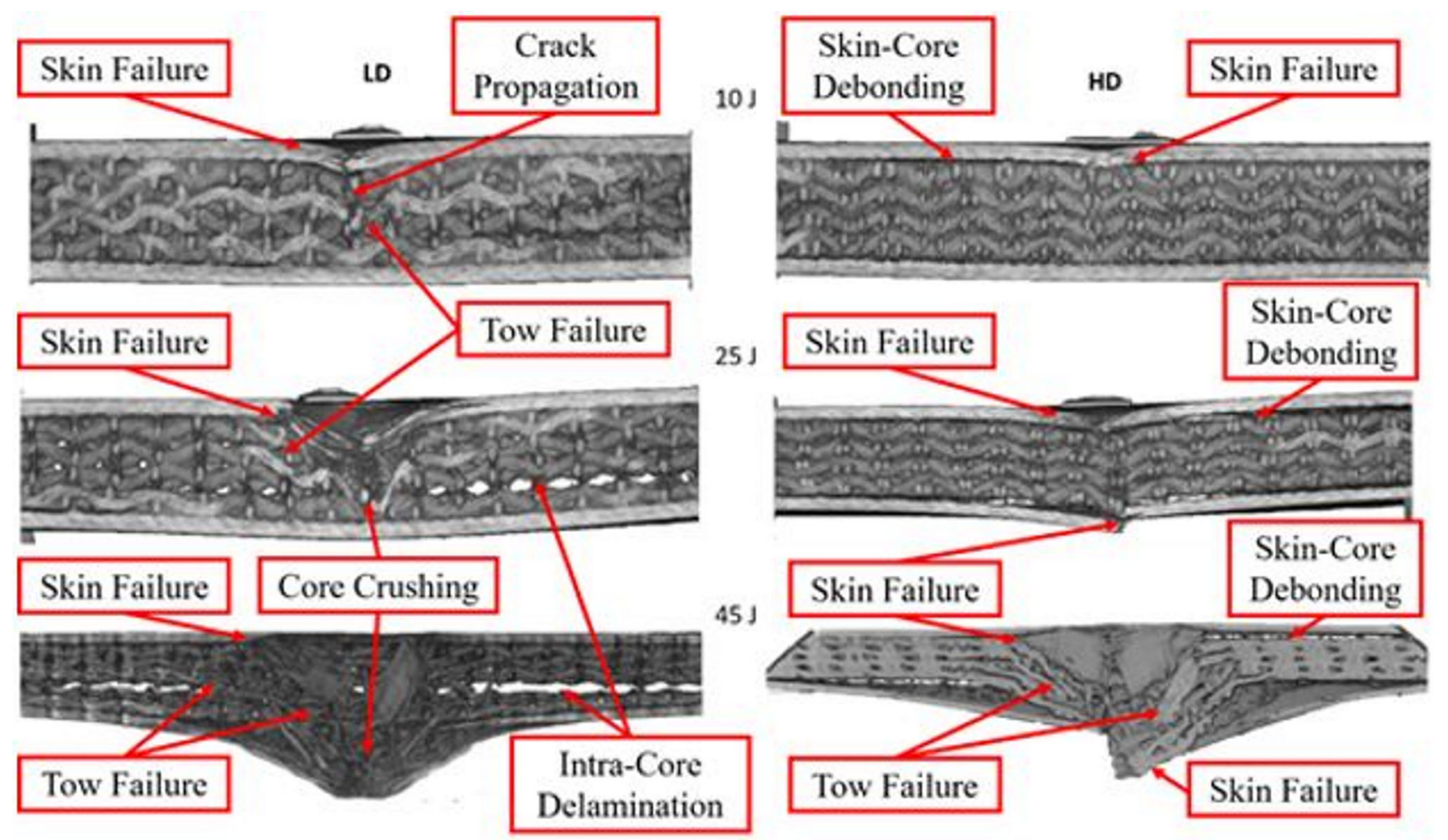

Fig. 11 CT scan results for HD_4P and LD_4P specimens impacted at 10-25-45 J (images at same scale as Fig. 3 and 4)

the propagation of tensile cracks at the bottom interface for the HD samples.

The observation for both LD_4P and HD_4P samples has been previously stated: increasing the thickness to six plies exposed both samples to less damage. The intra-core delamination of the LD_6P sample was accompanied by core crushing and failure of the top face sheet. The HD_6P sample revealed clear signs of face-sheet core debonding with failure of the top face sheet. As previously seen when the effect of variable impact energies was inspected, the failure modes remain consistent between different core thicknesses.

Figure 15 reveals the scans performed on the specimens with six plies impacted at 45 and $50 \mathrm{~J}$. The LD_6P samples at $45 \mathrm{~J}$ behave slightly different to the previous specimens of the same configuration, as in this case there is a clear sign of bottom skin failure with an intra-core delamination shifted toward the top portion of the core. On the other side, the HD_6P samples start to show signs of intra-core delamination (in the bottom portion of the core) with core crushing. As for 
the samples impacted at $50 \mathrm{~J}$, both LD and HD configurations suffer bottom skin damage and debonding (severe for the first) and high core crushing, but the latter shows no signs of intracore delamination. The deviation of the failure modes for both typologies from the ones observed in previous tests, can be attributed to the greater amounts of impact energy they were subjected to.

In order to investigate the planar extent of the internal damage and how it propagates through the core, Fig. 16 illustrates a top view of both LD_4P and HD_4P samples impacted at $45 \mathrm{~J}$. The first image shows the impacted area with an approximate estimation of the extension of the damage on the top face sheets while for the other images, the layers are virtually removed one by one, in an attempt to evaluate the extent of the damage of the samples. Despite the large amount of energy, the samples were subjected to, the different mechanisms are clear from the images. The assumption of the co-operation between the top skin and the core, in terms of energy absorption, is evident from the first image where the LD

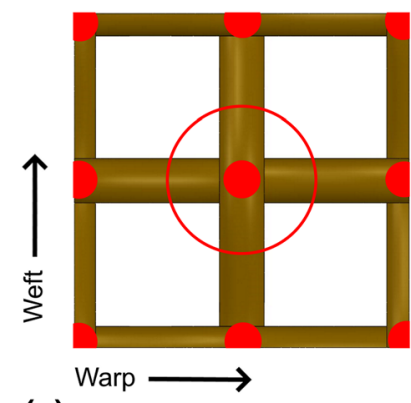

(a)

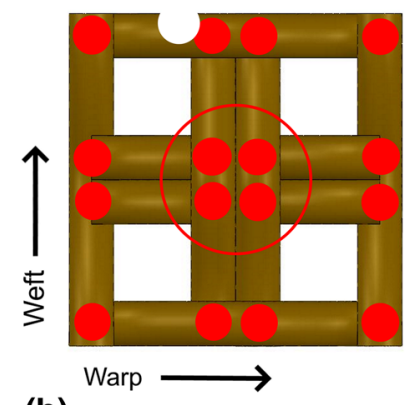

(b)

Fig. 12 Contact points of (a) LD typology and (b) HD typology typology shows a smaller damaged area as opposed to the HD one, with a reduction of approximately $17.5 \%$, as a result of the more elastic response of the LD configuration. The two subsequent images are congruent to the previous ones, showing highly distorted tows around the damaged area for the LD typology, while the HD configuration shows the total breakage of the grid due to the complete penetration of the impactor's tip. At the third layer the aforementioned failure modes become more noticeable; intra-core delamination is revealed between the LD typology's plies by an appearance of a curvature on the tows, while the HD typology endures the overall stiff response, with localized damage around the impacted area. At the fourth and final layer, the failure mechanism remained the same for the LD sample; however, the debonding at the core-face sheet interface occurred for the HD sample. It is important to highlight that the bottom skins of the samples were omitted from these images due to the lack of useful information that could be yielded, due to the large deformation and damage sustained (see Fig. 15).

From the experimental campaign it is possible to conclude that the difference in the impact response is due to the intrinsic geometric characteristic of the bi-grid structures that form the cores. In particular, the HD typology presents stiffer characteristics than that the LD one and this parameter affects the impact absorption mechanism. Indeed, when the samples are subjected to an impact event, a high-velocity impact wave is generated from the point of contact between the shuttle and the free surface and travels through the thickness as a compressive wave until it reaches the back end of the sample where it is reflected from the bottom skin and converts into a tensile wave. These periodic compressive and tensile stresses are dissipated more efficiently by the LD cores due to their more flexible response (given by the bending dominated mechanism of its unit cell) while for the more brittle HD cores these result in the

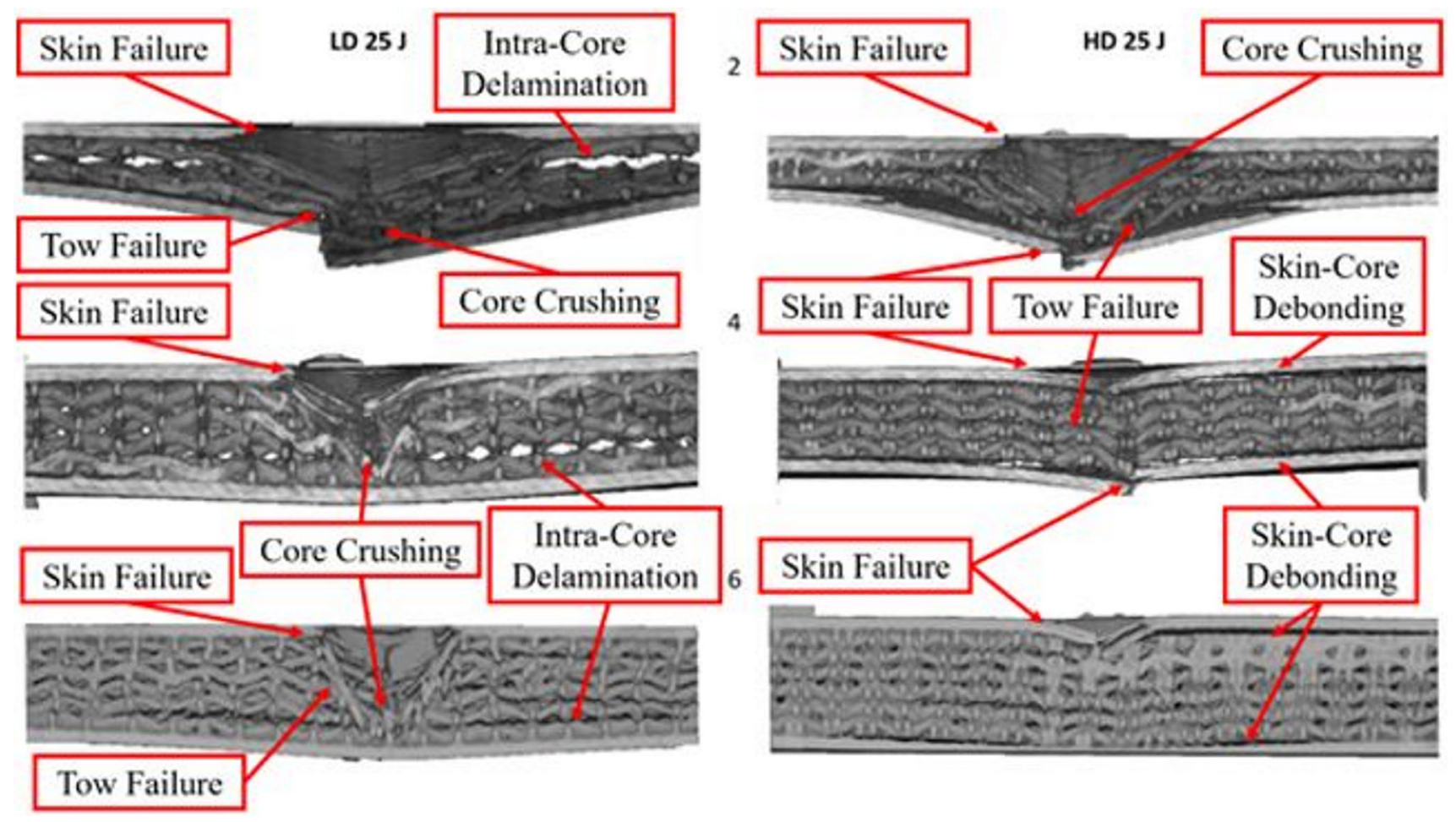

Fig. 13 CT scan results for 2-4-6 plies specimen impacted at $25 \mathrm{~J}$ (images at same scale as Fig. 6 and 7) 


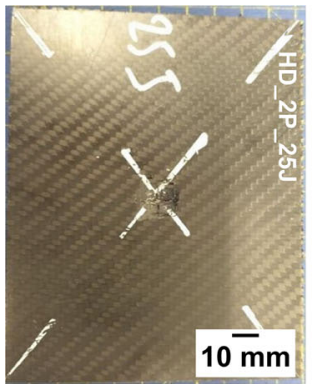

(a)

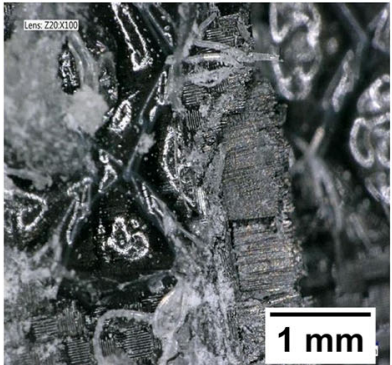

(e)

(b)
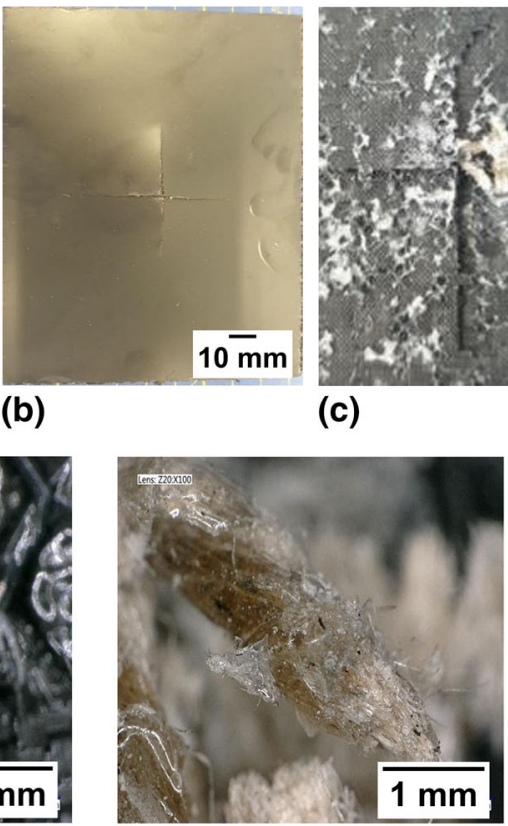

(f)

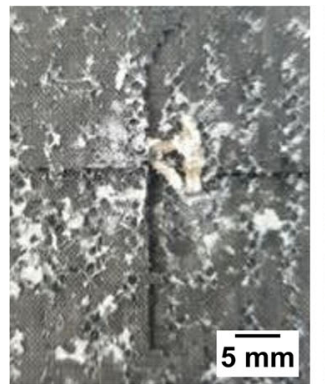

(c) (d)

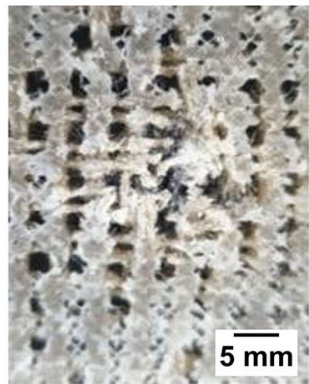

Fig. 14 Analysis of sample HD_2P impacted at $25 \mathrm{~J}$ : (a) post-impact top surface; (b) post-impact bottom surface; (c) surface of the bottom skin after the manual separation from the core; (d) bottom surface of the hemp core after manual separation from the skin; (e) Microscopy of the surface of the skin showing uniform distribution of adhesive on the carbon layer; (f) Microscopy of the surface of the core showing uniform distribution of the adhesive around the hemp yarns; (g) a fragment of the core still attached on the surface of the skin after the separation of the two parts of the sandwich
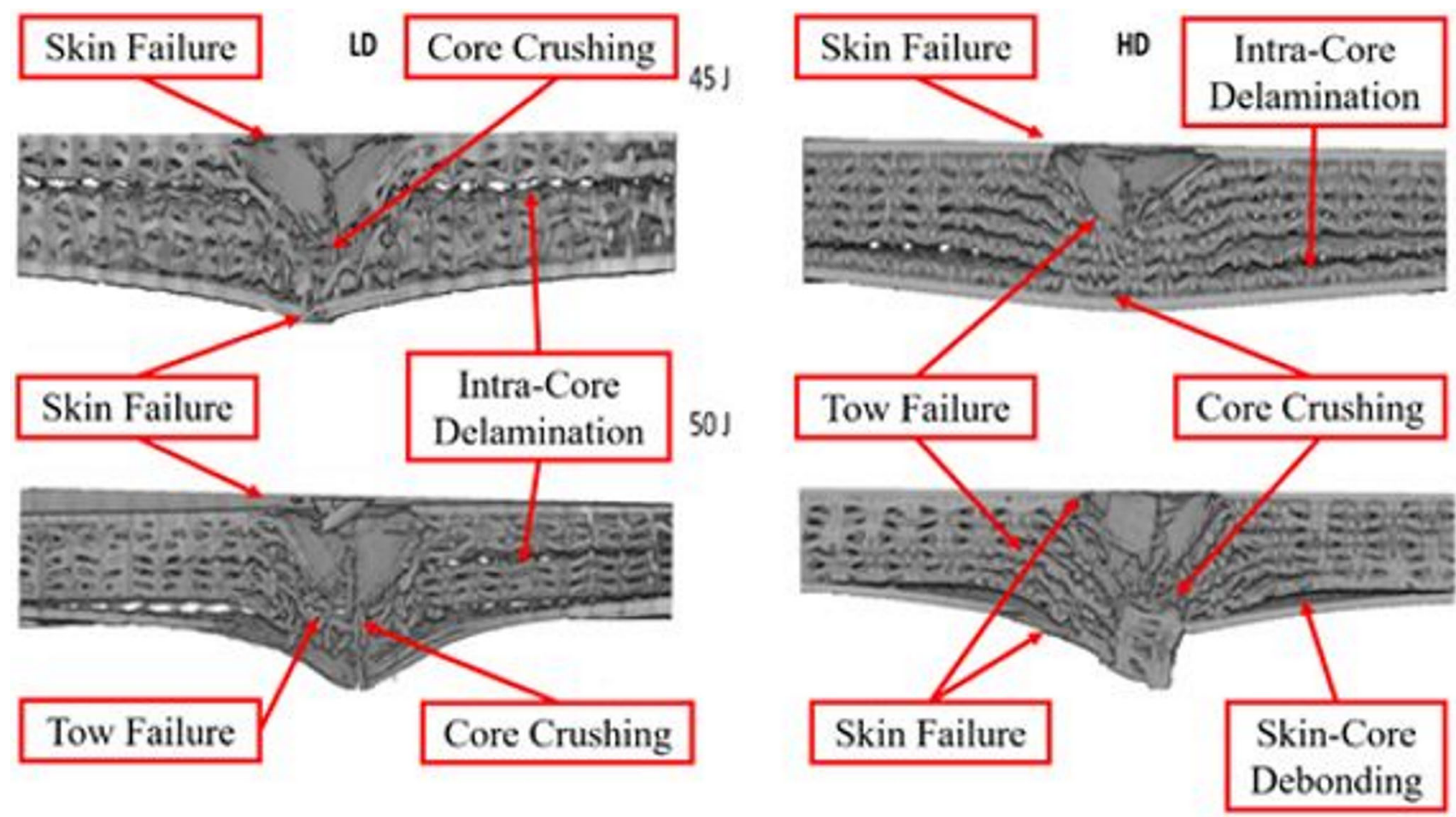

Fig. 15 CT scan results for HD_6P and LD_6P specimens impacted at 45-50 J (images at same scale as Fig. 9 and 10)

growth of cracks parallel to the lower interface (which is loaded in tension) that lead to the a localized cohesive failure at the interface between the skin and the core.

Figure 17 further justifies this assumption; the images shown are the midplanes of the two different typologies, focused around the impacted area and viewed from the top of the samples. It can be clearly seen that the LD typology has had a more flexible reaction to the impact test, with tows around the damaged area being distorted through a greater circular area. On the other side, the HD typology, due to its higher rigidity, 


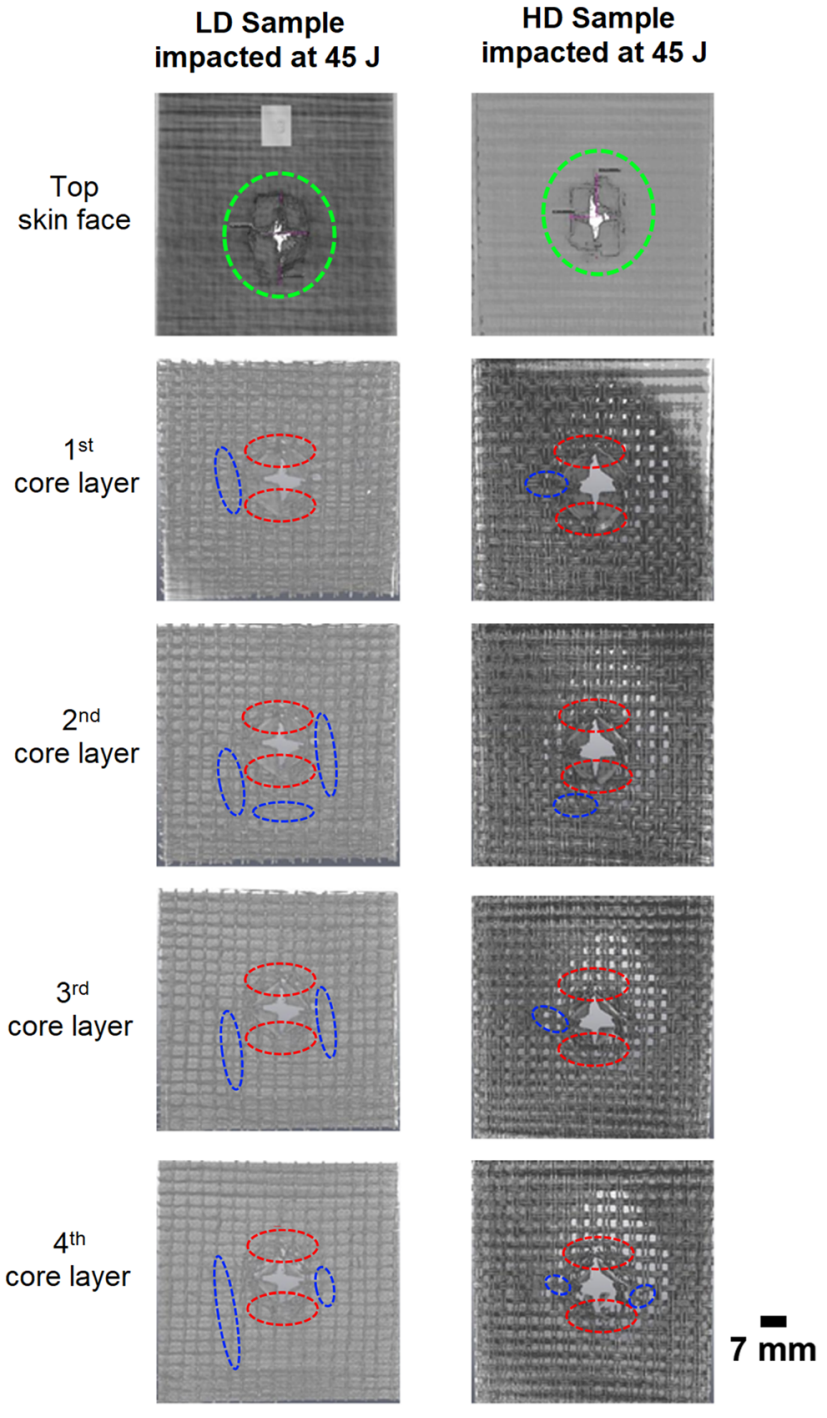

Fig. 16 Progressive top view of CT-scan images of (a) LD_4P and (b) HD_4P typologies impacted at $45 \mathrm{~J}$. Green circles indicate impact location, red circles tow failure and blue circles bent tows (Color figure online)

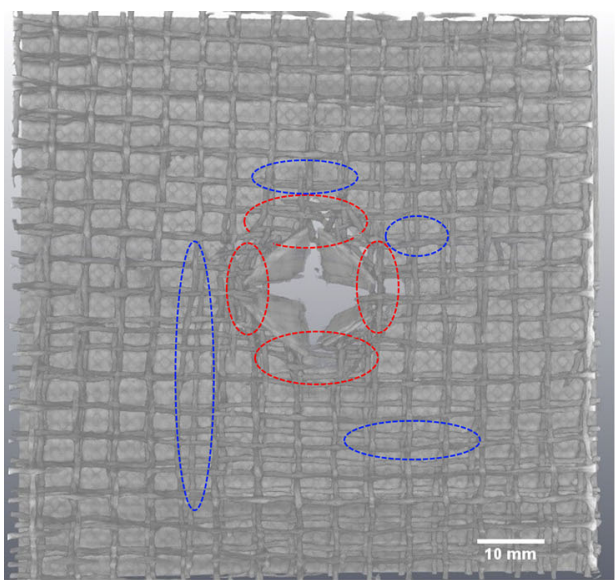

(a) presents a smaller damaged area, with tows close to the impacted area remaining undistorted keeping the reticular pattern of the core.

\subsection{Indentation Test}

Figure 18 reports typical specific indentation force-displacement curves referred to the indentation tests carried out on both LD_6P and HD_6P hemp core samples. The specific indentation force $\left(F_{W}\right)$ was defined by the ratio between the indentation force and the sample weight.

By comparing the curves, it is clear that the HD configuration responds almost elastically until the first peak force, while the LD_6P shows a change in the slope of the loading portion of the indentation curve, sign of larger deformations at the same loading conditions. Hence, a clear stiffer behavior is detected for the HD_6P sample in comparison with the LD_6P configuration, with the latter showing a reduction in the mean value of the specific peak force in comparison with the HD configuration.

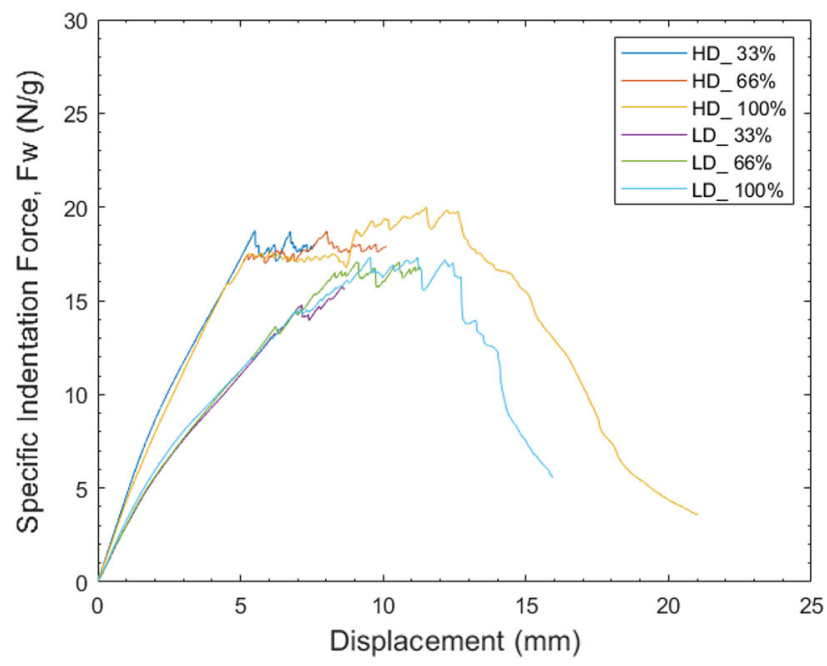

Fig. 18 Indentation test carried out on both LD_6P and HD_6P sample typologies

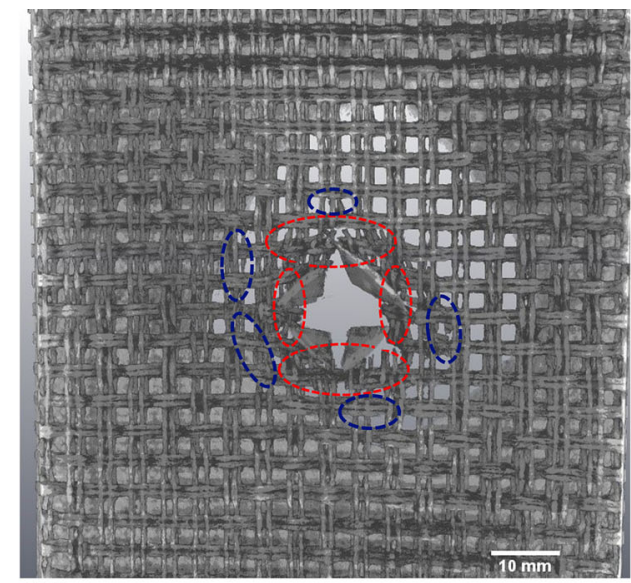

(b)

Fig. 17 Midplane view of (a) LD_4P specimen and (b) HD_4P sample (top view), when impacted at $45 \mathrm{~J}$. Red areas illustrate tow failures, whereas blue areas bent tows (Color figure online) 


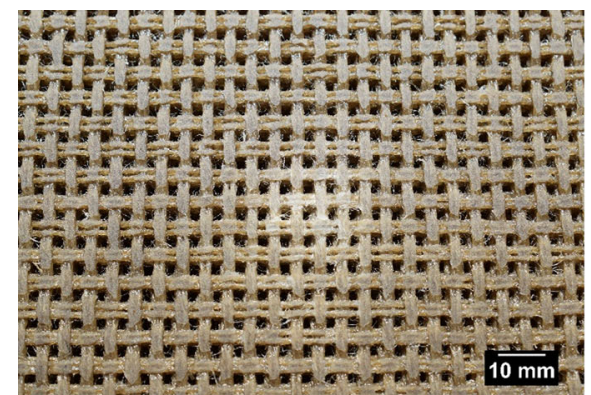

(a)

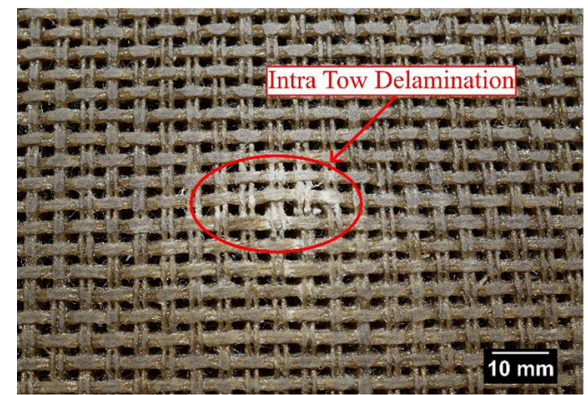

(c)

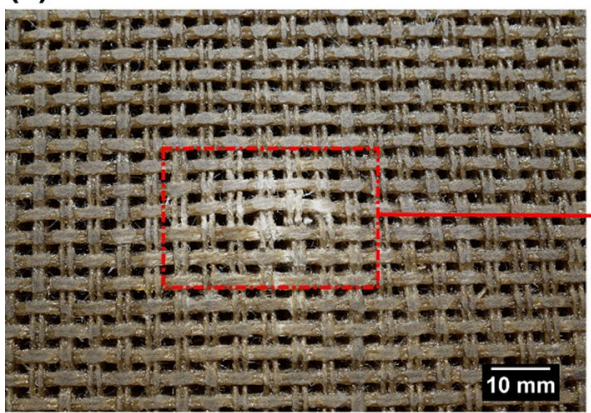

(e)

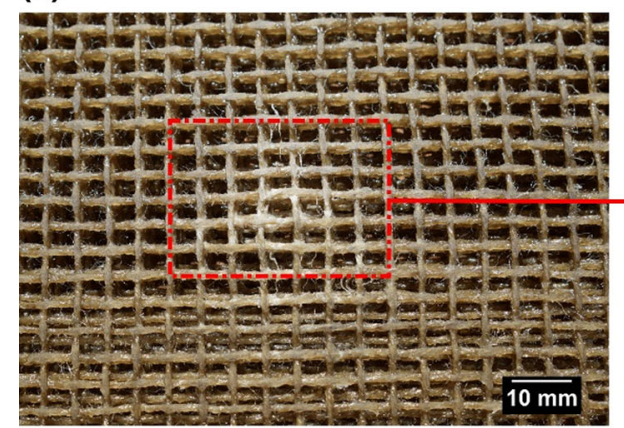

(f)

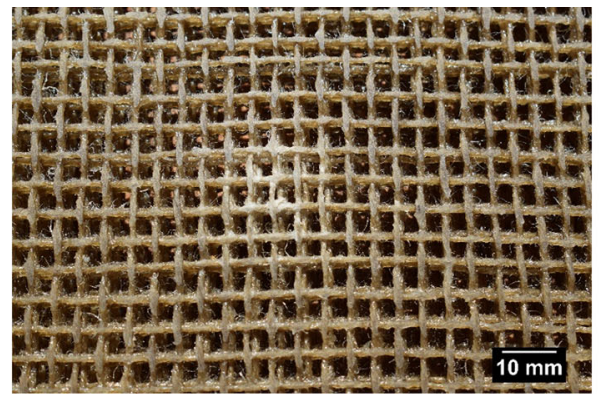

(b)

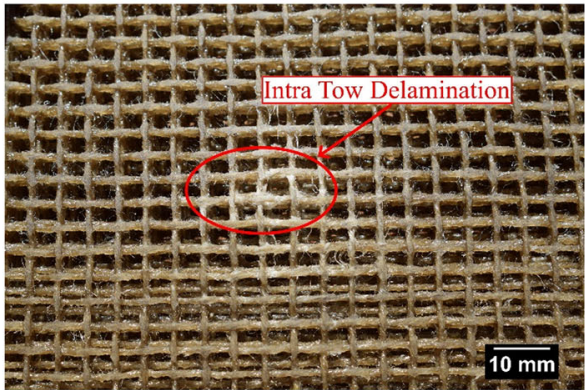

\section{(d)}

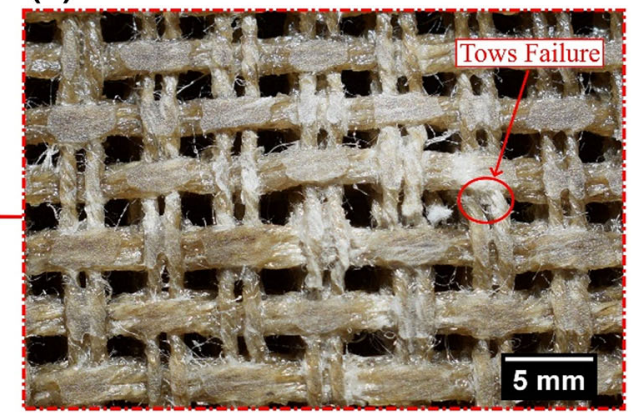

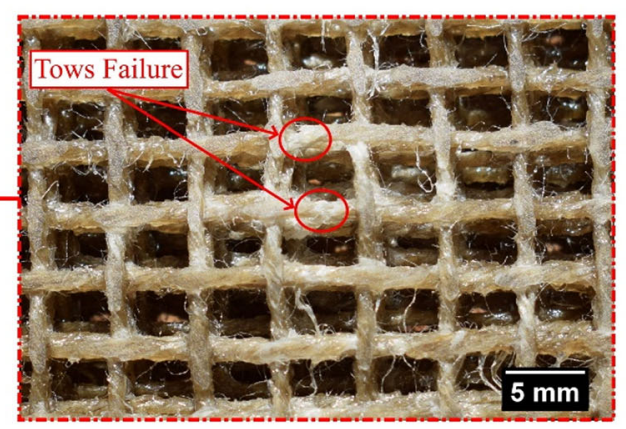

Fig. 19 Indented bottom surfaces: 33\% of the maximum displacement HD_6P sample (a) and LD_6P sample (b), 66\% HD_6P sample (c) and LD_6P sample (d), magnification of the damaged area of the 66\% indented samples HD_6P specimen (e) and LD_6P specimen (f)

It was observed a $12.8 \%$ reduction in the mean value of the specific indentation force of the LD configuration (mean value of $16.7 \mathrm{~N} / \mathrm{g}$ ) compared to the HD one (mean value of $19.1 \mathrm{~N} /$ g). At the same time, the LD_6P hemp core shows an increase of almost $30 \%$ of the displacement in correspondence of the peak load.

All these aspects lead to conclude that the LD_6P sample is affected by larger flexural deformations emphasized by the ductile behavior of the hemp fibers and the reduced areal density that characterizes the low density grid.
A visual inspection of the bottom surfaces of both sample typologies indented at $33 \%$ and $66 \%$ of the maximum displacement (Fig. 19), does not show a clear sign of core failure; however a clear damaged zone is detected in correspondence of the loading region.

A greater magnification of the samples' bottom surface (Fig. 19e and f), reveals the presence of broken hemp fibers as consequence of the flexural stresses that generate during the indentation test. Additional cracks propagate from the loading zone involving the superficial resin layer that covers the hemp tows; this aspect is attributed to the mechanical properties' 


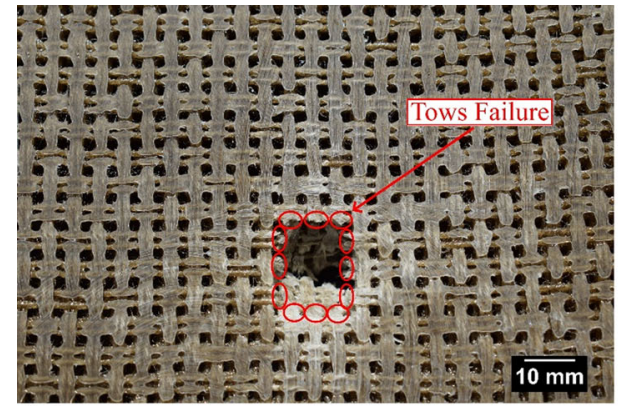

(a)

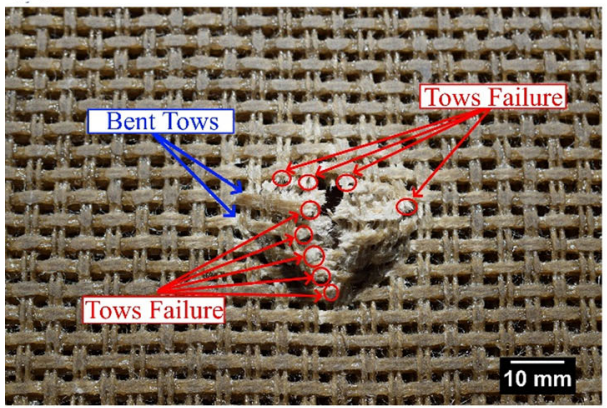

(c)

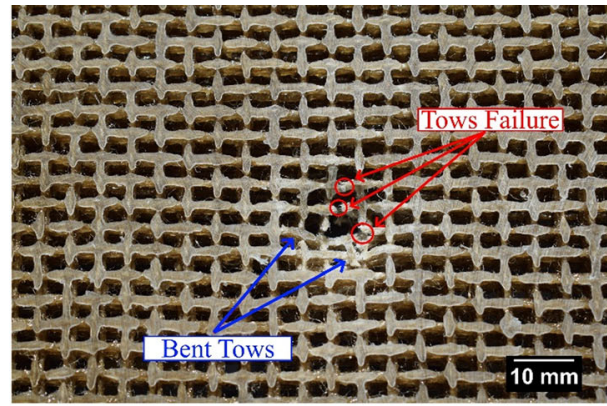

(b)

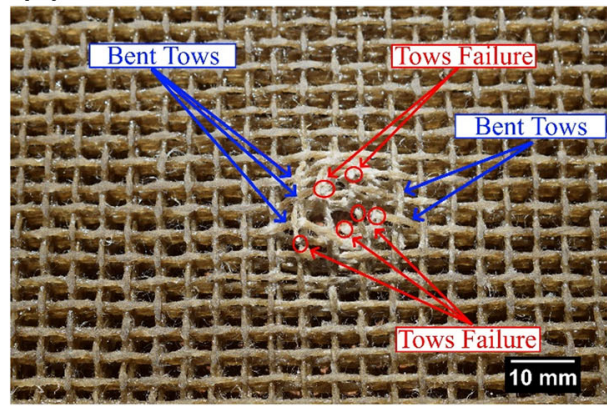

(d)

Fig. 20 Top view at penetration of (a) HD_6P and (b) LD_6P samples, bottom view at penetration of (c) HD_6P and (d) LD_6P samples

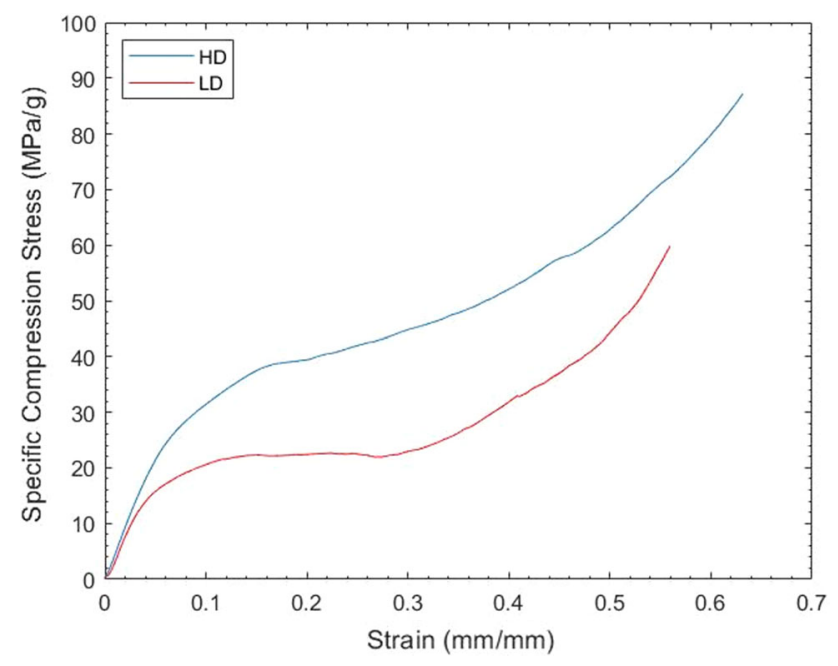

Fig. 21 Flatwise compression test curves carried out on both HD_6P and LD _6P sample typologies

mismatch between the brittle epoxy resin and the ductile hemp fibers causing fiber debonding and intra-tow delamination. Under these load conditions, it is possible to assert that even if both the sample typologies are partially damaged, they are still able to react to the applied loads.

The indentations carried out at penetration (Fig. 20) are the most representative, because these tests sustain the failure mechanisms observed from the impact tests and CT-Scan analysis. Both the LD_6P and HD_6P samples demonstrate clear sign of core failure, but as well as highlighted by the CTScan analysis, the areal density is able to influence the failure mechanism of these NF hemp core. The stiffer behavior that characterizes the HD sample leads to a severe core crushing with broken fibers in proximity of the indenter tip (Fig. 20a), while the typical diamond shape damage that characterizes composite materials failure is detected on the bottom surface (Fig. 20c) (Ref 11, 32, 33). The LD typology is characterized by a more elastic response showing bent fibers and a reduced indentation on the top surface in proximity of the indenter (Fig. 20b). Focusing the attention on the bottom surface, it is possible to observe broken fibers and an onset tows deformation (Fig. 20d) confirming what was already observed from the CT-Scan analyses (Fig. 16).

This behavior can be explained by observing that the LD configuration is characterized by a reduced number of core tows that leads to a smaller number of contact points between each layer that in turn allows for a larger flexural deformation if compared to the HD configuration. Furthermore, once the failure between the warp and weft tows connection occurs, thanks to the reduced areal density, a larger degree of freedom characterizes the LD hemp tows, as proved by the presence of in-plane bent fibers on the bottom surface of the enlarged hemp core structure (Fig. 20d).

\subsection{Compression Test}

Figure 21 represents the typical specific compression strength-strain curves of the flatwise compression test carried out on both LD_6P and HD_6P cores.

By comparing the curves, it is possible to observe that the HD_6P sample shows a higher compression strength in comparison with the LD_6P configuration. The trends also indicate that the HD_6P sample presents an almost purely brittle response; it is not possible to distinguish any plateau that would indicate the collapse of the core.

Analyzing the results, the LD sample shows a reduction in the compression strength and modulus per unit of weight, respectively, of almost 50\% and 37\% compared with the HD hemp core. These results can be justified by the different areal density that characterizes each layer of both core configurations. In detail, the good alignment of fibers in warp and weft 

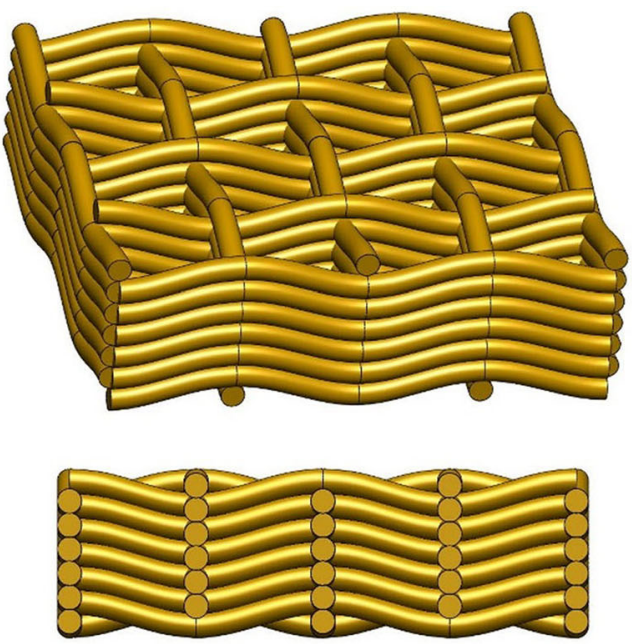

(a)
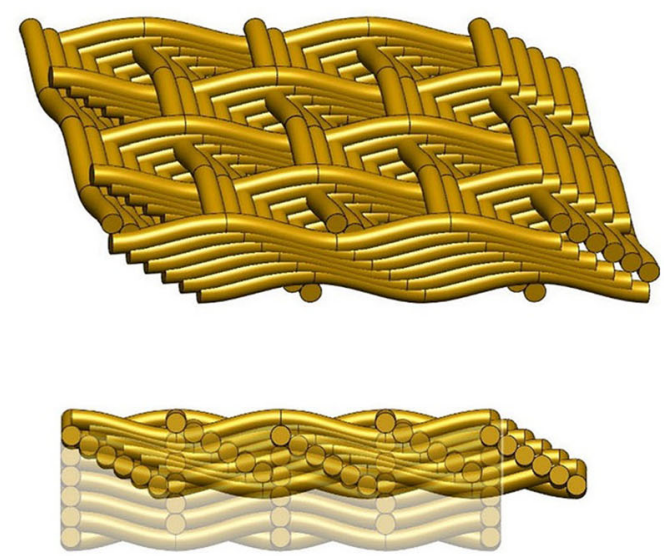

(b)

Fig. 22 Schematization of the LD_6P sample (a) before and (b) after the compression test

directions and the double number of hemp tows per unit of area of the HD configuration, guarantee improved compression properties in comparison with the LD one.

Analyzing the results from the tests, it appears clear that the mechanism of the core crushing represents one of the most important differences in the response of LD samples to out-ofplane loads. Indeed, it is thanks to this behavior that the material is able to accommodate larger deformations, absorbing more energy while preventing the brittle failure of the entire structure. This mechanism is generated by the presence of an instability within the unit cell of the LD samples which is schematized in Fig. 22, which shows the LD_6P hemp core before and after the compression test.

As it is possible to observe from this schematization, during the compression of the LD sample, each layer is affected by an in-plane sliding movement that generates interlaminar shear solicitations resulting in a core squeezing in both fabric directions with hemp tows that move from their original position to the fabric's mesh span.

This aspect is highlighted by the residual thickness evaluation as the LD_6P configuration shows a residual thickness which is 38\% lower than the HD_6P hemp core sample due to the more efficient compression mechanism given by the planar instability.

\section{Conclusions}

Natural fibers are an eco-friendly and sustainable resource for the manufacturing of cores for composite sandwich structures, and constitute a green alternative to traditional materials, such as foams or honeycombs. In this work, hemp fibers were manufactured using a custom continuous process and then arranged in a grid structure that enabled the formation of three-dimensional natural fiber cores. Two core typologies were studied by changing the geometry of the hemp bi-grid, obtaining LD and HD configurations $\left(0.33\right.$ and $0.63 \mathrm{~g} / \mathrm{cm}^{3}$, respectively) and several samples were manufactured with various thicknesses (between 2.84 and $8.16 \mathrm{~mm}$ ). The NF cores were subjected to indentation and compression tests and later bonded to CFRP face sheets and subjected to Low-Velocity Impact testing, so that their energy absorption and failure mechanisms could be investigated. The data post-processing of the experimental campaign revealed that the LD typology displayed ductile behavior, with a more elastic response and larger deformations when compared to the HD typology which revealed a stiffer, brittle behavior with more localized deformations. The LD typology also revealed a reduced compressive strength characterized by an out-of plane instability mechanism that is responsible of shear stresses that, in combination with the flexural solicitation, lead to severe intra-core delamination that affect this configuration when the impact energy goes above $10 \mathrm{~J}$. In terms of absorption energy at various impact energy levels, increasing the thickness of the samples leads to an increase in the energy absorbed, up to $+70 \%$ in the LD_6P typology, with the LD configuration proving to be more efficient at dissipate impact energy. The post-impact CT-Scan testing revealed the different failure mechanisms of the two typologies in out of plane loading conditions; the fewer contact points in the unit cell of the LD typology resulted in a more elastic deformations that lead to intra-core delamination at greater energy levels, whereas the stiff response of the HD typology resulted in debonding in the face sheet-core region. In conclusion, the suggested NF cores provide a high-tailorable solution for the manufacturing of sandwich panels for structural applications, showing a good combination of excellent energy absorption and potential weight savings given by the combination of carbon and hemp fibers, while also resulting in higher sustainability.

\section{Funding}

Open access funding provided by Università degli Studi di Napoli Federico II within the CRUI-CARE Agreement.

\section{Open Access}

This article is licensed under a Creative Commons Attribution 4.0 International License, which permits use, sharing, adaptation, distribution and reproduction in any medium or format, as long as you give appropriate credit to the original author(s) and the source, provide a link to the Creative Commons licence, and indicate if changes were made. The images or other third party material in this article are included in the article's Creative Commons licence, unless indicated otherwise in a credit line to the material. If 
material is not included in the article's Creative Commons licence and your intended use is not permitted by statutory regulation or exceeds the permitted use, you will need to obtain permission directly from the copyright holder. To view a copy of this licence, visit http://creativecommons.org/licenses/by/4.0/.

\section{References}

1. N.S. Fatima, G.S. Dhaliwal, and G. Newaz, Influence of Interfacial Adhesive on Impact and Post-impact Behaviors of CFRP/End-Grain Balsawood Sandwich Composites, Compos. Part B Eng., 2021, 212, p 108718

2. A.J. Turner, M. Al Rifaie, A. Mian, and R. Srinivasan, Low-Velocity Impact Behavior of Sandwich Structures with Additively Manufactured Polymer Lattice Cores, J. Mater. Eng. Perform., 2018, 27(5), p $2505-$ 2512

3. H. Norouzi and Y. Rostamiyan, Experimental and Numerical Study of Flatwise Compression Behavior of Carbon Fiber Composite Sandwich Panels with New Lattice Cores, Constr. Build. Mater, 2015, 100, p 22 30

4. S.C. Sharma, M. Krishna, H.N. Narasimha Murthy, M. Sathyamoorthy, and D. Bhattacharya, Fatigue Studies of Polyurethane Sandwich Structures, J. Mater. Eng. Perform., 2004, 13(5), p 637-641

5. H. Yang, H. Lei, and G. Lu, Crashworthiness of Circular Fiber Reinforced Plastic Tubes Filled with Composite Skeletons/Aluminum Foam under Drop-Weight Impact Loading, Thin-Walled Struct., 2021, 160, p 107380

6. O.T. Thomsen, Sandwich Materials for Wind Turbine Blades-Present and Future, J. Sandw. Struct. Mater., 2009, 11(1), p 7-26

7. H.N.G. Wadley, Multifunctional Periodic Cellular Metals, Philos Trans. R. Soc. A Math. Phys. Eng. Sci., 2006, 364(1838), p 31-68

8. F. Zhu, G. Lu, D. Ruan, and Z. Wang, Plastic Deformation, Failure and Energy Absorption of Sandwich Structures with Metallic Cellular Cores, Int. J. Prot. Struct., 2010, 1(4), p 507-541

9. A.M. Abou-Ali, O. Al-Ketan, R. Rowshan, and R. Abu Al-Rub, Mechanical Response of 3D Printed Bending-Dominated LigamentBased Triply Periodic Cellular Polymeric Solids, J. Mater. Eng. Perform., 2019, 28(4), p 2316-2326

10. J. Xiong, L. Feng, R. Ghosh, H. Wu, L. Wu, L. Ma, and A. Vaziri, Fabrication and Mechanical Behavior of Carbon Fiber Composite Sandwich Cylindrical Shells with Corrugated Cores, Compos. Struct., 2016, 156, p 307-319

11. F. Pinto, L. Boccarusso, D. De Fazio, S. Cuomo, M. Durante, and M. Meo, Carbon/Hemp Bio-Hybrid Composites: Effects of the Stacking Sequence on Flexural, Damping and Impact Properties, Compos. Struct., 2020, 242, p 112148

12. C. Santulli, Mechanical and Impact Damage Analysis on Carbon/ Natural Fibers Hybrid Composites: A Review, Materials (Basel), 2019, 12(3), p 285-299

13. Y. Swolfs, I. Verpoest, and L. Gorbatikh, Recent Advances in FibreHybrid Composites: Materials Selection, Opportunities and Applications, Int. Mater. Rev., 2019, 64(4), p 181-215

14. S. Kumar, D. Zindani, and S. Bhowmik, Investigation of Mechanical and Viscoelastic Properties of Flax- and Ramie-Reinforced Green Composites for Orthopedic Implants, J. Mater. Eng. Perform., 2020, 29(5), p 3161-3171

15. A. Astarita, L. Boccarusso, M. Durante, A. Viscusi, R. Sansone, and L. Carrino, Study of the Production of a Metallic Coating on Natural Fiber Composite through the Cold Spray Technique, J. Mater. Eng. Perform., 2018, 27, p 739-750
16. M.Y.M. Zuhri, Z.W. Guan, and W.J. Cantwell, The Mechanical Properties of Natural Fibre Based Honeycomb Core Materials, Compos. Part B Eng., 2014, 58, p 1-9

17. G. Cicala, G. Recca, L. Oliveri, Y. Perikleous, F. Scarpa, C. Lira, A. Lorato, D.J. Grube, and G. Ziegmann, Hexachiral Truss-Core with Twisted Hemp Yarns: Out-of-Plane Shear Properties, Compos. Struct., 2012, 94(12), p 3556-3562

18. J.P. Vitale, G. Francucci, J. Xiong, and A. Stocchi, Failure Mode Maps of Natural and Synthetic Fiber Reinforced Composite Sandwich Panels, Compos. Part A Appl. Sci. Manuf., 2017, 94, p 217-225

19. A. Sarwar, Z. Mahboob, R. Zdero, and H. Bougherara, Mechanical Characterization of a New Kevlar/Flax/Epoxy Hybrid Composite in a Sandwich Structure, Polym. Test., 2020, 90, p 106680

20. Y. Chen, S. Hou, K. Fu, X. Han, and L. Ye, Low-Velocity Impact Response of Composite Sandwich Structures: Modelling and Experiment, Compos. Struct., 2017, 168, p 322-334

21. S.N.A. Safri, M.T.H. Sultan, M. Jawaid, and K. Jayakrishna, Impact Behaviour of Hybrid Composites for Structural Applications: A Review, Compos. Part B Eng., 2018, 133, p 112-121

22. S. Li, R. Hu, J. Cheng, and Y. Hu, Compressive Effect of Jute Fiber Corrugated Lattice Sandwich Structure, J. Reinf. Plast. Compos., 2020, 39(5-6), p 209-218

23. L. Boccarusso, L. Carrino, M. Durante, A. Formisano, A. Langella, and F.M.C. Minutolo, Hemp Fabric/Epoxy Composites Manufactured by Infusion Process: Improvement of Fire Properties Promoted by Ammonium Polyphosphate, Compos. Part B Eng., 2016, 89, p 117126

24. S. Yin, H. Chen, Y. Wu, Y. Li, and J. Xu, Introducing Composite Lattice Core Sandwich Structure as an Alternative Proposal for Engine Hood, Compos. Struct., 2018, 201, p 131-140

25. D. De Fazio, L. Boccarusso, S. Cuomo, M. Durante, F.M.C. Minutolo, M. Meo, K. Myronidis, and F. Pinto, Impact Behaviour of a New Hemp/Carbon Sandwich Structure, Proc. CIRP, 2021, 99, p 432-436

26. L. Boccarusso, M. Durante, and A. Langella, Lightweight Hemp/BioEpoxy Grid Structure Manufactured by a New Continuous Process, Compos. Part B Eng., 2018, 146, p 165-175

27. G. Petrone, S. Rao, S. De Rosa, B.R. Mace, F. Franco, and D. Bhattacharyya, Behaviour of Fibre-Reinforced Honeycomb Core under Low Velocity Impact Loading, Compos. Struct., 2013, 100, p 356-362

28. A. De Luca, F. Caputo, Z. Sharif Khodaei, and M.H. Aliabadi, Damage Characterization of Composite Plates under Low Velocity Impact Using Ultrasonic Guided Waves, Compos. Part B Eng., 2018, 138, p $168-180$

29. T. Anderson and E. Madenci, Experimental Investigation of LowVelocity Impact Characteristics of Sandwich Composites, Compos. Struct., 2000, 50(3), p 239-247

30. R.A.W. Mines, C.M. Worrall, and A.G. Gibson, The Static and Impact Behaviour of Polymer Composite Sandwich Beams, Composites, 1994, 25(2), p 95-110

31. V. Birman, Dynamic Wrinkling in Sandwich Beams, Compos. Part $B$ Eng., 2004, 35(6-8), p 665-672

32. F. Rizzo, F. Pinto, and M. Meo, 3D Bio-Inspired Hierarchical Discontinuous CFRP with Enhanced Ductility, Compos. Struct., 2019, 226, p 111202

33. R. Park and J. Jang, Impact Behavior of Aramid Fiber/Glass Fiber Hybrid Composites: The Effect of Stacking Sequence, Polym. Compos., 2001, 22(1), p 80-89

Publisher's Note Springer Nature remains neutral with regard to jurisdictional claims in published maps and institutional affiliations. 